\title{
Long noncoding RNA LINC00649 functions as a microRNA-432-5p sponge to facilitate tumourigenesis in colorectal cancer by upregulating HDGF
}

\author{
JUNJIE BAO* ${ }^{*}$ XIAOKAI BI* ${ }^{*}$ JINGBO WANG and XIAOQIANG LI \\ Department of General Surgery, The First People's Hospital of Chongqing Liangjiang, Chongqing 401121, P.R. China
}

Received February 1, 2021; Accepted June 28, 2021

DOI: $10.3892 / \mathrm{mmr} .2022 .12620$

\begin{abstract}
Long intergenic nonprotein coding RNA 649 (LINC00649) is a functional regulator in acute myeloid leukaemia. However, the contribution of LINC00649 in colorectal cancer (CRC) has yet to be confirmed. Accordingly, the present investigation was devoted to exploring the detailed functions of LINC00649 and reveal the mechanisms underlying the LINC00649-induced promotion of CRC progression. LINC00649 expression in CRC was investigated by reverse transcription-quantitative PCR. Knockdown of LINC00649 was achieved using small interfering RNAs or short hairpin RNA, followed by functional experiments. The binding between LINC00649 and microRNA (miR)-432-5p was predicted by a bioinformatics tool, and corroborated by luciferase reporter assay and RNA immunoprecipitation. In the present study, LINC00649 was expressed at a high level in CRC. The aberrant expression of LINC00649 exhibited an inverse association with CRC patient prognosis. Functionally, the downregulation of LINC00649 exerted anticarcinogenic activities in CRC by decreasing cell proliferation, migration, and invasion and inducing cell apoptosis. Furthermore, the growth of CRC cells in vivo was attenuated after LINC00649 deficiency. Mechanistically, LINC00649 functioned as a competitive endogenous RNA by competitively binding to miR-432-5p in CRC cells, inducing an increase in hepatoma-derived growth factor (HDGF). Ultimately, functional rescue experiments highlighted that the exogenous introduction of miR-432-5p inhibitor or HDGF overexpression plasmid partially abated the inhibitory effects of LINC00649 silencing.
\end{abstract}

Correspondence to: Professor Jingbo Wang or Professor Xiaoqiang Li, Department of General Surgery, The First People's Hospital of Chongqing Liangjiang, 199 Renxing Road, Chongqing 401121, P.R. China

E-mail: dr_jingbowang@126.com

E-mail: lixiaoq_general@163.com

${ }^{*}$ Contributed equally

Key words: long noncoding RNA, colorectal cancer, competitive endogenous RNA pathway, microRNA sponge
In conclusion, LINC00649 promoted the aggressiveness of CRC cells by adjusting the miR-432-5p/HDGF axis. Thus, the LINC00649/miR-432-5p/HDGF pathway may be a promising target for CRC therapy.

\section{Introduction}

Colorectal cancer (CRC), a frequent malignant tumour of the digestive tract, ranks third in terms of morbidity and is the second most common cause of cancer-related deaths worldwide (1). Annually, 1.3 million novel CRC cases occur globally, which cause $>0.6$ million deaths (2). Curative resection along with chemotherapy can be applied in a small proportion of patients, whereas a great proportion of patients with CRC are diagnosed at late stage or suffer from post-surgical diseases such as recurrence or distant metastasis (3). Although marked improvements have been identified in CRC therapy, the therapeutic efficacy for patients at advanced stages has not distinctly improved (4). Notably, the major causes for worse clinical outcomes are delayed diagnosis, tumour relapse and distant metastasis (5). Therefore, elucidating the mechanisms underlying $\mathrm{CRC}$ pathogenesis is urgently required in order to find novel putative targets for diagnosis and treatment.

Long noncoding RNAs (lncRNAs) are a type of RNA transcript with $>200$ nucleotides that have lost protein-coding capacity (6). Although lncRNAs were previously regarded as useless, they have been revealed to produce considerable effects in numerous biological behaviours, including cell differentiation, the cell cycle, cytogenetic processes and epigenetic modification (7). Notably, lncRNAs have been described to perform important regulatory actions in promoting and maintaining tumour initiation and progression (8). To date, a growing number of lncRNAs have been confirmed to be dysregulated in CRC and closely associated with malignant characteristics (9). The differentially expressed lncRNAs play carcinogenic or anti-oncogenic actions and consequently participate in the control of CRC progression (10).

MicroRNAs (miRNAs) area family of non-protein-coding RNAs with $\sim 17-23$ nucleotides (11). They have been reported to exhibit crucial functions in posttranscriptional regulation of genes via binding to their 3'untranslated regions (3'UTRs), triggering transcriptional silencing or mRNA reduction (12). The contribution of miRNAs in CRC has received widespread attention in the past decade, and 
extensive miRNAs have been confirmed to be implicated in CRC progression by exerting tumour-promoting or tumour-inhibiting roles (13-15). Specifically, the competitive endogenous RNA (ceRNA) theory has been recognized and has indicated that lncRNAs execute a 'sponge-like' activity on certain miRNAs, thereby abolishing or weakening the modulatory activities of miRNAs on their targets (16). Subsequently, comprehending the dysregulated lncRNAs in CRC and clarifying their working mechanisms may be instrumental in the development of attractive targets for cancer diagnosis and therapy.

Long intergenic nonprotein coding RNA 649 (LINC00649) has been identified as a functional regulator in acute myeloid leukaemia (17). In addition, the lncRNA has been validated as a hub lncRNA that may be strongly correlated with colorectal carcinogenesis and progression (18). However, the detailed roles of LINC00649 in CRC have not been fully confirmed. Therefore, the specific roles of LINC00649 in CRC and the related mechanisms were explored.

\section{Materials and methods}

Patient tissues. The present research was authorized (approv al no. EC-FPHCQLND-20150815) by the Ethics Committee of The First People's Hospital of Chongqing Liangjiang (Chongqing, China) and was implemented in strict accordance with the Declaration of Helsinki. Written informed consent was provided by all participants. CRC tissues were acquired from 52 patients with CRC ( 30 male patients and 22 female patients; age range, 41-72 years) at The First People's Hospital of Chongqing Liangjiang (Chongqing, China) between June 2015 and January 2016. Adjacent normal tissues were acquired $3 \mathrm{~cm}$ away from tumour tissues. All patients included in the present study underwent a form of anticancer treatment before the operation. Additionally, patients who were diagnosed with other types of human cancer were excluded. All patient tissues were preserved in liquid nitrogen.

Cell culture. A normal human colon epithelium cell line FHC was acquired from the American Type Culture Collection (ATCC), and cultured in DMEM:F12 medium (cat. no. 11320033; Gibco; Thermo Fisher Scientific, Inc.) with $25 \mathrm{mM}$ HEPES, $10 \mathrm{ng} / \mathrm{ml}$ cholera toxin, $0.005 \mathrm{mg} / \mathrm{ml}$ insulin, $0.005 \mathrm{mg} / \mathrm{ml}$ transferrin, $100 \mathrm{ng} / \mathrm{ml}$ hydrocortisone, $20 \mathrm{ng} / \mathrm{ml}$ human recombinant epidermal growth factor and $10 \%$ foetal bovine serum (FBS; Gibco; Thermo Fisher Scientific Inc.). HCT 116 (ATCC), a CRC cell line, was cultured in McCoy's 5a medium (cat. no. 16600108; Gibco; Thermo Fisher Scientific, Inc.) and 10\% FBS. Another two CRC cell lines, SW480 and SW620 (both from the National Collection of Authenticated Cell Cultures; Shanghai, China), were maintained in $10 \%$ FBS-supplemented L-15 medium (cat. no. 11415114; Gibco; Thermo Fisher Scientific Inc.). All these cells were cultured at $37^{\circ} \mathrm{C}$ and $5 \% \mathrm{CO}_{2}$.

Transfection. Specific small interfering RNAs (siRNAs) against LINC00649 (si-LINC00649), non-targeting negative control siRNA (si-NC), miR-432-5p mimic, NC miRNA mimic (miR-NC), miR-432-5p inhibitor (anti-miR-432-5p) and miRNA inhibitor control (anti-miR-NC) were all constructed
Table I. Sequences of siRNAs, miRNA oligonucleotides and shRNAs.

\begin{tabular}{ll}
\hline Type & \multicolumn{1}{c}{ Sequences $\left(5^{\prime} \rightarrow 3^{\prime}\right)$} \\
\hline si-LINC00649\#1 & TAGATGTTATTGACAAAATAAGC \\
si-LINC00649\#2 & AAGTTAAAGCTTCCAAAAATAGA \\
si-NC & CACGATAAGACAATGTATTT \\
miR-432-5p & UCUUGGAGUAGGUCAUUGGGUGG \\
mimic & \\
miR-NC & CGAUCGCAUCAGCAUCGAUUGC \\
miR-432-5p & CCACCCAAUHACCUACUCCAAGA \\
inhibitor & \\
anti-miR-NC & UGAGCUGCAUAGAGUAGUGAUUA \\
sh-LINC00649 & CCGGTAGATGTTATTGACAAAATAA \\
& GCCTCGAGGCTTATTTTGTCAATAA \\
sh-NC & CATCTATTTTTG \\
& CCGGCACGATAAGACAATGTATTTC \\
& TCGAGAAATACATTGTCTTATCGTG \\
& TTTTTG
\end{tabular}

si-, small interfering; miRNA or miR, microRNA; sh-, short hairpin; LINC00649, long intergenic nonprotein coding RNA 649; NC, negative control.

by Shanghai GenePharma Co., Ltd. The sequences are presented in Table I. The pcDNA3.1-hepatoma-derived growth factor (pc-HDGF) plasmid was obtained from Shanghai GenePharma Co., Ltd. First, cells were inoculated into 6-well plates and cultured to $\sim 60-80 \%$ density. Next, the cells were transfected with siRNAs (100 pmol), mimic (100 pmol), miRNA inhibitor (100 pmol) or plasmid $(4 \mu \mathrm{g})$ by applying Lipofectamine $^{\circledast} 2000$ (Invitrogen; Thermo Fisher Scientific, Inc.) according to the manufacturer's instructions. After incubation for $6 \mathrm{~h}$ with transfection reagent at $37^{\circ} \mathrm{C}$, the medium was replaced with fresh medium. Reverse transcription-quantitative (RT-q)PCR, flow cytometry and Transwell migration and invasion assays were carried out at $48 \mathrm{~h}$ post-transfection. After incubation for $24 \mathrm{~h}$, Cell Counting Kit-8 (CCK-8) assay was conducted.

RT-qPCR. Small RNA was extracted from cultured cells or tissues utilizing RNAiso for Small RNA and reverse-transcribed utilizing a Mir-X miRNA First-Strand Synthesis Kit (both from Takara Biotechnology Co., Ltd.) according to the manufacturer's instructions. For quantification of miR-432-5p, qPCR was executed with Mir-X miRNA RT-qPCR TB Green ${ }^{\circledR}$ Kit (Takara Biotechnology Co., Ltd.) according to the manufacturer's instructions, and the data were normalized to U6 small nuclear RNA. The thermocycling conditions were as follows: $95^{\circ} \mathrm{C}$ for $10 \mathrm{sec} ; 40$ cycles at $95^{\circ} \mathrm{C}$ for $5 \mathrm{sec}$ and $60^{\circ} \mathrm{C}$ for $20 \mathrm{sec}$; and $95^{\circ} \mathrm{C}$ for $60 \mathrm{sec}, 55^{\circ} \mathrm{C}$ for $30 \mathrm{sec}$ and $95^{\circ} \mathrm{C}$ for $30 \mathrm{sec}$.

Tissues or cells were immersed in $1 \mathrm{ml}$ TRIzol (Invitrogen; Thermo Fisher Scientific, Inc.), after which they were incubated at room temperature for $5 \mathrm{~min}$. Prior to being centrifuged at $1,000 \mathrm{x} \mathrm{g}$ at $4^{\circ} \mathrm{C}$ for $15 \mathrm{~min}, 200 \mu \mathrm{l}$ chloroform was 
supplemented into the Eppendorf tube and rigorous shaking was executed for $15 \mathrm{sec}$. The supernatant fluid was transferred to a novel Eppendorf, followed by the addition of $500 \mu \mathrm{l}$ isopropanol. After centrifugation at $1,000 \mathrm{x} \mathrm{g}$ at $4^{\circ} \mathrm{C}$ for $10 \mathrm{~min}$, the supernatant fluid was discarded, and the precipitant was washed with $1 \mathrm{ml} 75 \%$ alcohol and centrifuged at $1,000 \mathrm{xg}$ at $4^{\circ} \mathrm{C}$ for $5 \mathrm{~min}$. The RNA was collected and dissolved in RNA-free water. Then, the isolated RNA was reverse transcribed into cDNA by PrimeScript ${ }^{\mathrm{TM}}$ RT reagent Kit (Takara Biotechnology Co., Ltd.) according to the manufacturer's instructions. To assess LINC00649 and HDGF expression, TB Green ${ }^{\circledR}$ Premix Ex Taq ${ }^{\mathrm{TM}}$ (Takara Biotechnology Co., Ltd.) was applied for PCR amplification. The thermocycling conditions were as follows: Initial denaturation at $95^{\circ} \mathrm{C}$ for $30 \mathrm{sec}$, followed by 40 cycles at $95^{\circ} \mathrm{C}$ for $3 \mathrm{sec}, 60^{\circ} \mathrm{C}$ for $30 \mathrm{sec}$ and $72^{\circ} \mathrm{C}$ for $30 \mathrm{sec}$. GAPDH served as the internal reference for LINC00649 and HDGF. The $2^{-\Delta \Delta C q}$ method (19) was used to analyse the expression of genes.

The primers were designed as follows: LINC00649 forward, 5'-CTTGCAGTTTGATCTCAGACTGC-3' and reverse, 5'-ACCACGAGACTATATCCCACACC-3'; HDGF forward, 5'-GTGACGGTGATAAGAAGGGGAAT-3' and reverse, 5'-TTCAACGCTCCTTTCTCGTTCT-3'; GAPDH forward, 5'-AGTCAACGGATTTGGTCGTATTG-3' and reverse, 5'-AAACCATGTAGTTGAGGTCAATGAA-3'; U6 forward, 5'-CTCGCTTCGGCAGCACA-3' and reverse, 5'-AACGCT TCACGAATTTGCGT-3'; miR-497-5p forward, 5'-TCGGCA GGCAGCAGCACACUG-3'; miR-432-5p forward, 5'-TCG GCAGGUCUUGGAGUAGG-3'; miR-28-3p forward, 5'-TCG GCAGGAGGUCCUCGAG-3'; miR-195-5p forward, 5'-TCG GCAGGUAGCAGCACAG-3'; miR-15b-5p forward, 5'-TCG GCAGGUAGCAGCACAUCA-3'. The universal reverse sequence for miRNA was 5'-CACTCAACTGGTGTCGTG GA-3' (reverse).

CCK-8 assay. Each well of 96-well plates was supplemented with $100 \mu \mathrm{l}$ cell suspension containing $2 \times 10^{3}$ transfected cells. Subsequently, $10 \mu$ l CCK-8 reagent (Nanjing KeyGen Biotech Co., Ltd.) was added, and the culture plates were incubated at $37^{\circ} \mathrm{C}$ for $2 \mathrm{~h}$. The absorbance was measured with a microplate reader at a wavelength of $450 \mathrm{~nm}$. The assay was performed for three consecutive days, and the obtained results were used for growth curve construction.

Flow cytometric analysis for apoptosis. Two days following cell injection, $0.25 \%$ trypsin was employed for cell harvest $\left(2 \times 10^{6}\right)$. Phosphate-buffered saline (PBS) was pre-cooled at $4^{\circ} \mathrm{C}$ and used to wash the collected cells. In accordance with the instructions of the Annexin V-FITC Apoptosis Detection Kit (Beyotime Institute of Biotechnology), the obtained cells were resuspended in $195 \mu \mathrm{l}$ Annexin V-FITC, followed by mixing with $5 \mu \mathrm{l}$ Annexin V-FITC and $10 \mu \mathrm{l}$ propidium iodide. Subsequently, the cells were cultured at $25^{\circ} \mathrm{C}$ for $15 \mathrm{~min}$ and continually maintained in darkness. The early + late apoptotic cells were detected by a flow cytometer (FACScan ${ }^{\mathrm{TM}}$; BD Biosciences) and analysed with CellQuest software v.2.9 (BD Biosciences).

Transwell cellular migration and invasion assays. The migratory and invasive capacities of CRC cells were evaluated employing a Transwell chamber $(8-\mu \mathrm{m}$ pore size; BD Biosciences). For the invasion assay, Matrigel (BD Biosciences) was used to precoat the lower surface of the upper chamber at $37^{\circ} \mathrm{C}$ for $2 \mathrm{~h}$, whereas a migration assay was implemented without this step. For both assays, $5 \times 10^{4}$ transfected cells resuspended in FBS-free culture medium (McCoy's 5a medium for HCT116, L-15 medium for SW480) were seeded into the upper chambers. In the lower chambers, $600 \mu \mathrm{l}$ complete medium mixed with $20 \%$ FBS was plated and functioned as the chemoattractant. Cells were cultured at $37^{\circ} \mathrm{C}$ for one day, after which the non-migrated or non-invasive cells were removed from the upper face of the membrane. The migrated and invasive cells were fixed with ethanol at room temperature for $15 \mathrm{~min}$ and staining with $0.1 \%$ crystal violet at room temperature for $30 \mathrm{~min}$. After washing with PBS, the number of stained cells was observed under a light microscope (magnification, x200; Olympus Corporation).

Tumour xenograft in nude mice. Short hairpin RNA (shRNA) for LINC00649 (sh-LINC00649) and NC shRNA (sh-NC) were synthesized by Shanghai GenePharma Co., Ltd. A 2nd lentiviral system was used in the production of lentiviruses. The sequences are presented in Table I. The shRNAs were cloned into a lentivirus vector prior to being transduced into 293T cells (National Collection of Authenticated Cell Cultures) together with psPAX2 and pMD2.G. A total of $30 \mu \mathrm{g}$ plasmids were used in lentivirus packaging, and the ratio of lentiviral plasmid: psPAX2: pMD2.G was 2:1:1. After $6 \mathrm{~h}$ of cultivation at $37^{\circ} \mathrm{C}$, the medium was replaced with fresh $10 \%$ FBS-supplemented DMEM with $1 \%$ glutamax, $1 \%$ non-essential amino acids and $1 \%$ sodium pyruvate solution (all purchased from Gibco; Thermo Fisher Scientific, Inc.). Following 2 days of incubation, the lentiviruses carrying either sh-LINC00649 or sh-NC were harvested and utilized to infect HCT 116 cells with the multiplicity of infection (MOI) 5. The stable cell line was screened out by treatment with $2 \mu \mathrm{g} / \mathrm{ml}$ puromycin, and maintained with $1 \mu \mathrm{g} / \mathrm{ml}$ puromycin.

The animal study was performed with the approval (appr oval no. IACUC-FPHCQLND-20190601) of the Institutional Animal Care and Use Committee of The First People's Hospital of Chongqing Liangjiang (Chongqing, China). A total of $6 \mathrm{BALB} / \mathrm{c}$ nude mice (male; age, 4-6 weeks; mean weight, $20.12 \mathrm{~g}$ ) were supplied by Vital River Laboratory Animal Technology Co., Ltd. The mice were housed under specific pathogen-free conditions at $25^{\circ} \mathrm{C}$ and $50 \%$ humidity, with a 10/14 $\mathrm{h}$ light/dark cycle and ad libitum access to food and water. A total of $2 \times 10^{6} \mathrm{HCT} 116$ cells stably overexpressing sh-LINC00649 or sh-NC were resuspended in $100 \mu \mathrm{l}$ PBS and subcutaneously inoculated into the flank of mice. The width and length of the subcutaneous tumours were recorded weekly. The tumour volume was calculated with the equation: Volume $=0.50 \mathrm{x}$ length $\mathrm{x}$ width ${ }^{2}$. On the 28 th day, the mice were euthanized by means of cervical dislocation, and tumour xenografts were resected for tumour weighing.

Bioinformatic prediction and luciferase reporter assay. starBase 3.0 (http://starbase.sysu.edu.cn/) was employed to identify potential target miRNA of LINC00649. The putative targets of miR-432-5p were searched by applying TargetScan (version 7.2; http://www.targetscan.org/) and starBase 3.0. The 
Cancer Genome Atlas (TCGA; https://portal.gdc.cancer.gov/) database (20) was employed to analyse LINC00649 expression in CRC (TCGA-COAD and TCGA-READ Projects.).

Fragments of LINC00649 and HDGF carrying the wild-type (wt) miR-432-5p binding sequences were synthesized by Shanghai GenePharma Co., Ltd. After inserting into the pmirGLO reporter vector (Promega Corporation), the recombinant reporter plasmids were named LINC00649-wt and HDGF-wt. Similarly, the LINC00649-mutant (mut) and HDGF-mut were constructed in a similar manner. For the reporter assay, the reporter plasmids alongside miR-432-5p mimic or miR-NC were transfected into CRC cells using Lipofectamine 2000 (Invitrogen; Thermo Fisher Scientific, Inc.), followed by $48 \mathrm{~h}$ of culture at $37^{\circ} \mathrm{C}$ in an atmosphere containing $5 \% \mathrm{CO}_{2}$. Transfected cells were immersed in $100 \mu \mathrm{l}$ Passive Lysis Buffer provided from the Dual-Luciferase Reporter Assay kit (Promega Corporation) according to the manufacturer's instructions. The luminometer tubes were loaded with $50 \mu \mathrm{l}$ LAR II that was supplemented with $10 \mu 1$ cell lysis buffer. After the measurement of firefly luciferase activity, $50 \mu \mathrm{l}$ L Stop \& GLoR Reagent (Promega Corporation) was added, followed by the detection of Renilla luciferase activity. Renilla luciferase activity was used as the reference.

Subcellular fractionation analysis. The Cytoplasmic \& Nuclear RNA Purification Kit (Norgen Biotek Corp.) was applied for subcellular fractionation according to the manufacturer's instructions. After total RNA extraction from nuclear and cytoplasmic fractions, RT-qPCR was carried out to measure the relative amount of LINC00649 in both fractions. GAPDH and U6 functioned as normalizing cytoplasmic and nuclear controls, respectively.

RNA immunoprecipitation (RIP). The binding of LINC00649 and miR-432-5p to the Argonaute-2 (Ago2) protein was explored with a RIP assay, which was carried out employing a Magna RIP RNA-Binding Protein Immunoprecipitation Kit (cat. no. 17-700; MilliporeSigma) according to the manufacturer's instructions. CRC cells were immersed in RIP lysis buffer (MilliporeSigma) to obtain whole cell extracts. A 10\% cell extract was used as the input, and the other parts were treated with RIP buffer containing magnetic beads that were conjugated with human anti-Ago2 (1:500; cat. no. ab32381; Abcam) or anti-IgG antibodies (1:5,000; included in the kit mentioned above) at $4^{\circ} \mathrm{C}$ overnight. The input served as the positive control, while IgG was used as the negative control. The magnetic beads were harvested and probed with Proteinase K (Beyotime Institute of Biotechnology) at $55^{\circ} \mathrm{C}$ for $30 \mathrm{~min}$ with vibration to remove the protein. The immunoprecipitated RNA was examined with RT-qPCR to determine LINC00649 and miR-432-5p enrichment, and the RT-qPCR was performed according to the manufacturer's (Takara Biotechnology Co., Ltd.) instructions as outlined above.

Western blotting. Transfected cells were washed three times with precooled PBS, and RIPA buffer (Nanjing KeyGen Biotech Co., Ltd.) was added to isolate total protein. Next, a BCA Protein Assay Kit was adopted for protein quantification.
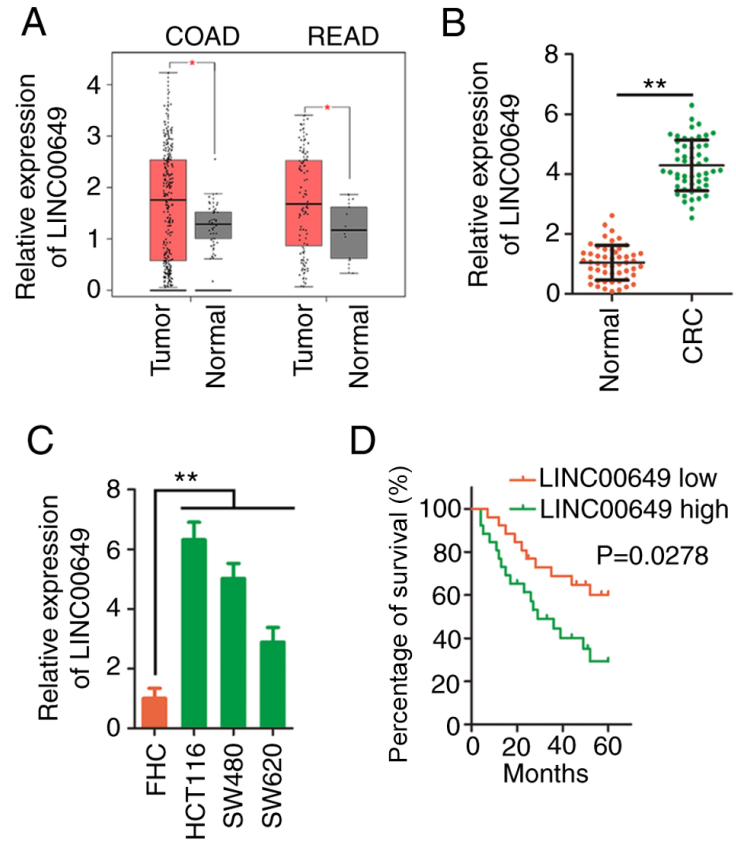

D

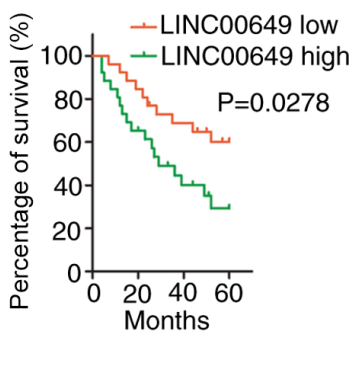

Figure 1. LINC00649 is overexpressed in CRC and presents an inverse relationship with patient prognosis. (A) The expression status of LINC00649 in COAD and READ was determined utilizing The Cancer Genome Atlas. (B) LINC00649 expression in CRC tissues was quantified. (C) Reverse transcription-quantitative PCR was performed to assess LINC00649 levels in three CRC cell lines. (D) The association of the expression level of LINC00649 with overall survival in patients with CRC was explored by Kaplan-Meier method. ${ }^{*} \mathrm{P}<0.05$ and ${ }^{* *} \mathrm{P}<0.01$. LINC00649, long intergenic nonprotein coding RNA 649; CRC, colorectal cancer; COAD, colon adenocarcinoma; READ, rectum adenocarcinoma.

Proteins (30 $\mu \mathrm{g} / \mathrm{lane})$ were loaded and electrophoresed on a $10 \%$ SDS-PAGE, followed by transferring to PVDF membranes and blocking the membranes at room temperature for $2 \mathrm{~h}$ with $5 \%$ non-fat milk powder. Subsequent to overnight incubation at $4^{\circ} \mathrm{C}$ with primary antibodies specifically targeting HDGF (cat. no. ab128921; 1:1,000) or GAPDH (cat. no. ab128915; 1:1,000; both from Abcam), the membranes were probed with a horseradish peroxidase (HRP)-conjugated secondary antibody (cat. no. ab205718; 1:5,000; Abcam) at room temperature for $2 \mathrm{~h}$. The protein signals were detected with an ECL detection kit (Nanjing KeyGen Biotech Co., Ltd.) and images were captured with ChemiDoc ${ }^{\mathrm{TM}}$ XRS+imaging system (Bio-Rad Laboratories, Inc.). Densitometry was performed using Quantity One software (version 4.6.6; Bio Rad Laboratories, Inc.).

Statistical analysis. The mean \pm standard deviation (SD) values of three independent replicates were employed to present the experimental data. The Kaplan-Meier method estimated the survival curves, which were then analysed with log-rank testing. Both paired and unpaired Student's t-tests were applied to detect comparisons between two groups. The differences between multiple groups were analysed by one-way analysis of variance (ANOVA), and Tukey's post hoc test was implemented following ANOVA. Pearson's correlation analysis was conducted to compare the correlation between the expression of LINC00649, miR-432-5p and HDGF. P $<0.05$ was considered to indicate a statistically significant difference. 
A

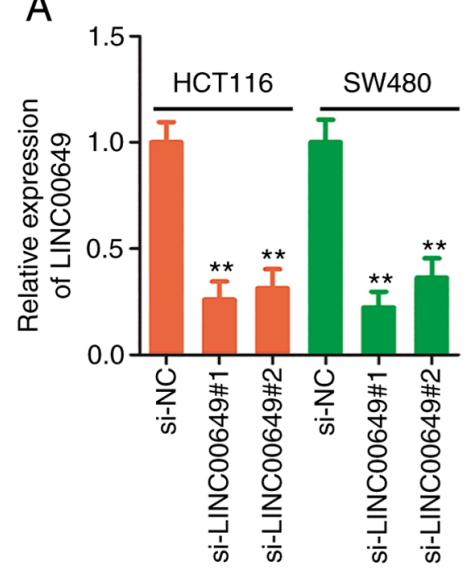

C
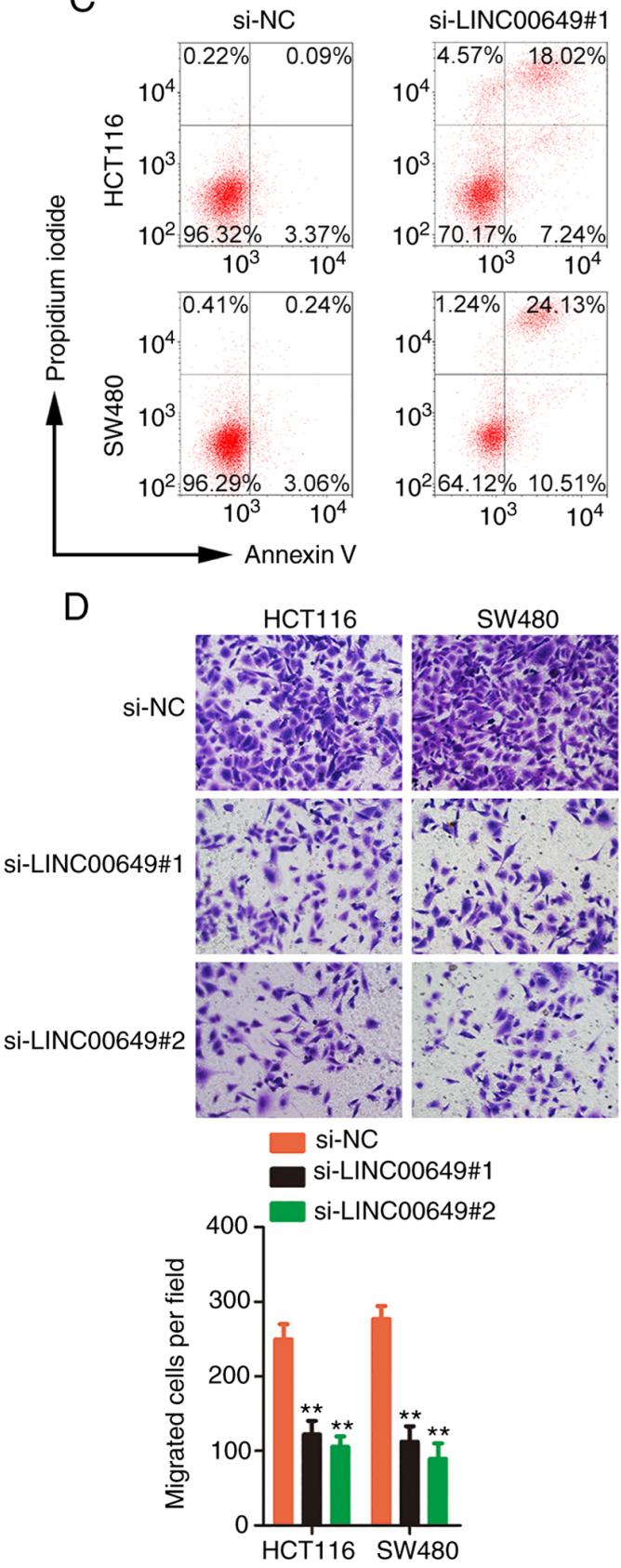

$\mathrm{B} \quad \rightarrow$ si-NC

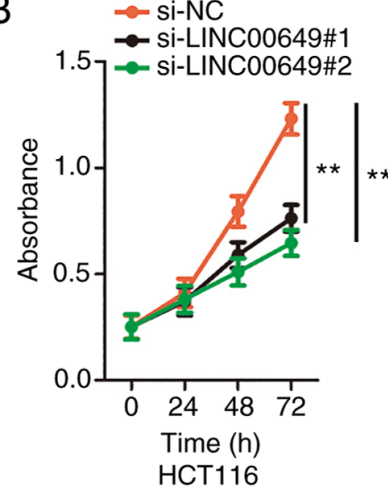

HCT116

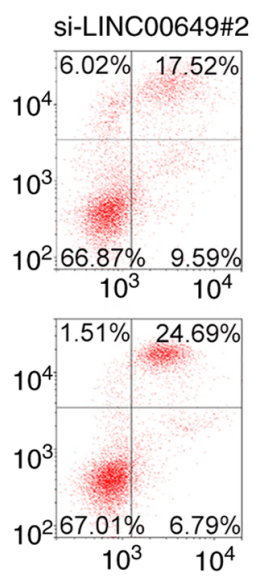

$\mathrm{E}$
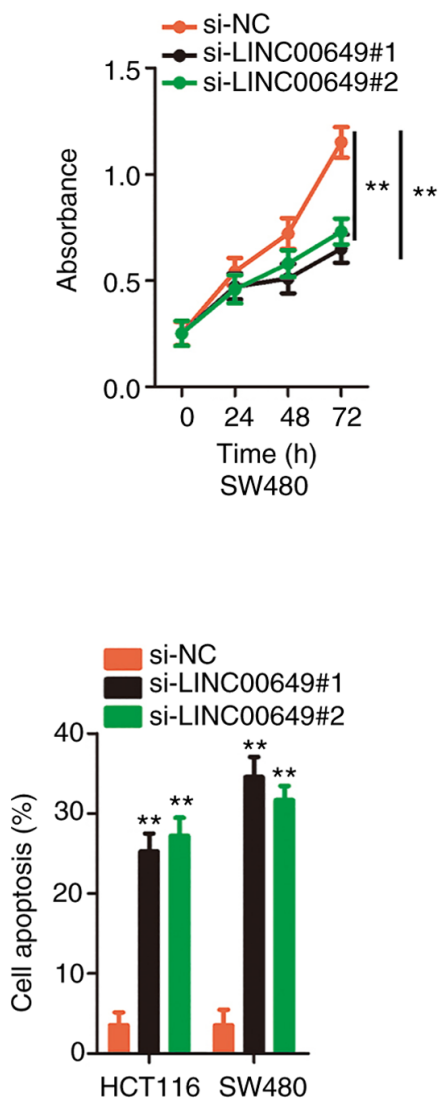
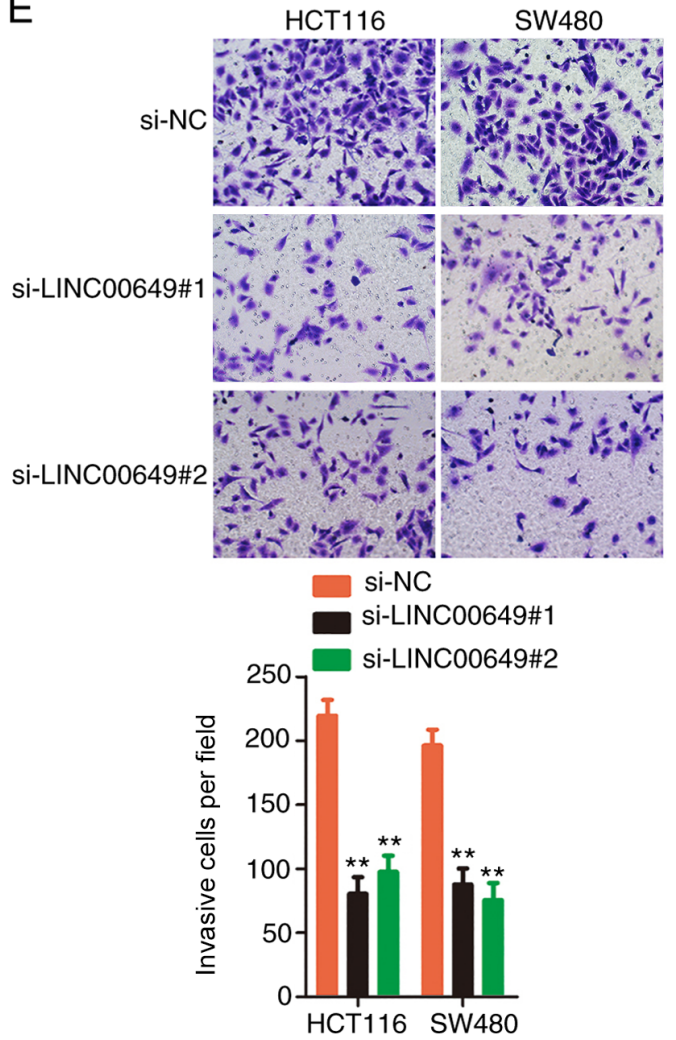

Figure 2. Silencing of LINC00649 restricts the malignant behaviours of CRC cells. (A) LINC00649 silencing efficiencies in CRC cells by si-LINC00649 were assessed by reverse transcription-quantitative PCR. (B) Cell proliferation was determined after the silencing of LINC00649. (C) The influence of si-LINC00649 on CRC cell apoptosis was assessed by flow cytometric analysis. (D and E) Transwell cellular migration and invasion assays were performed to evaluate the motility in LINC00649-deficient CRC cells (magnification, $\mathrm{x} 200$ ). ${ }^{* *} \mathrm{P}<0.01$ vs. si-NC. LINC00649, long intergenic nonprotein coding RNA 649; $\mathrm{CRC}$, colorectal cancer; si-, small interfering; $\mathrm{NC}$, negative control. 

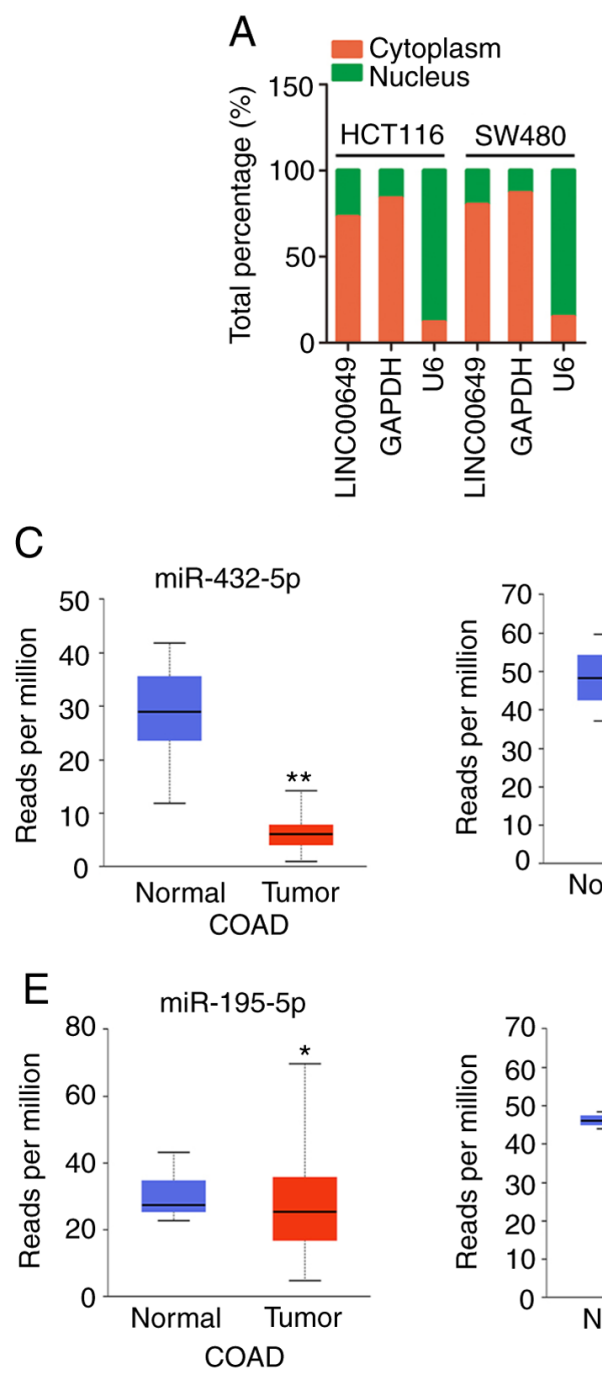
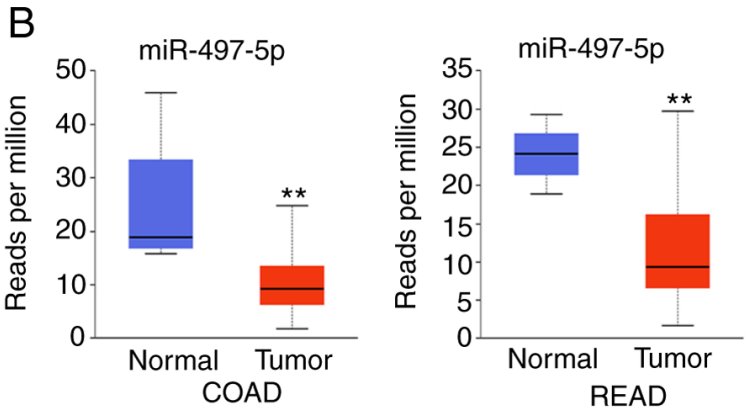

$\mathrm{D}$
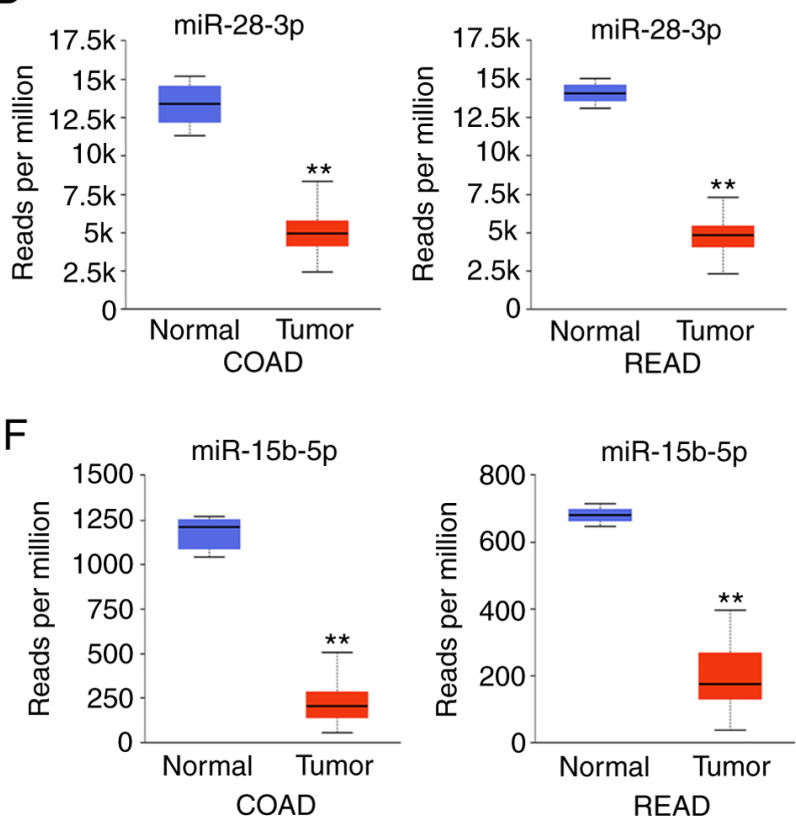

Figure 3. Localization of LINC00649 in CRC and expression of its putative targets. (A) Subcellular fractionation analysis was conducted and verified that LINC00649 was mostly located in CRC cell cytoplasm. Expression profiles of (B) miR-497-5p, (C) miR-432-5p, (D) miR-28-3p, (E) miR-195-5p and (F) miR-15b-5p in COAD and READ were determined in The Cancer Genome Atlas database. * $\mathrm{P}<0.05$ and ${ }^{* *} \mathrm{P}<0.01$ vs. Normal. LINC00649, long intergenic nonprotein coding RNA 649; CRC, colorectal cancer; miR, microRNA; COAD, colon adenocarcinoma; READ, rectum adenocarcinoma.

\section{Results}

LINC00649 is overexpressed in CRC. First, LINC00649 expression in CRC was analysed using a dataset from TCGA. As compared with normal tissues, LINC00649 was upregulated in colon adenocarcinoma (COAD) and rectum adenocarcinoma (READ) (Fig. 1A). Additionally, LINC00649 was detected in the cohort of the present study. LINC00649 was highly expressed in CRC tissues compared with adjacent normal tissues (Fig. 1B). Moreover, the three assessed CRC cell lines overexpressed LINC00649 compared with FHC (Fig. 1C). Next, utilizing the median value of LINC00649 expression in CRC tissues as the cut-off point, all subjects were subdivided into LINC00649-low or LINC00649-high expression groups. Survival analysis was performed, and the results revealed that a high expression level of LINC00649 was associated with shorter overall survival (Fig. 1D). Therefore, overexpressed LINC00649 was negatively associated with the prognosis of patients with CRC.
Silencing of LINC00649 impairs the malignant behaviours of CRC cells. The HCT 116 and SW480 cell lines expressed the highest expression of LINC00649 and accordingly, they were applied in subsequent research. To understand the detailed functions of LINC00649 in CRC progression, two siRNAs targeting LINC00649 were used to efficiently knock down LINC00649 expression (Fig. 2A). Then, CCK-8 assays confirmed that proliferative activity was hindered in CRC cells after the silencing of LINC00649 (Fig. 2B). Flow cytometric analysis also revealed that transfection with si-LINC00649 led to the promotion of CRC cell apoptosis (Fig. 2C). Furthermore, loss of LINC00649 expression suppressed the migratory (Fig. 2D) and invasive (Fig. 2E) properties of CRC cells. Briefly, these results confirmed the oncogenic actions of LINC00649 in CRC cells.

LINC00649 acts as a ceRNA by sponging miR-432-5p in CRC cells. To elucidate the molecular events of LINC00649, subcellular fractionation analysis was implemented to reveal the localization of LINC00649 in CRC cells. LINC00649 was 

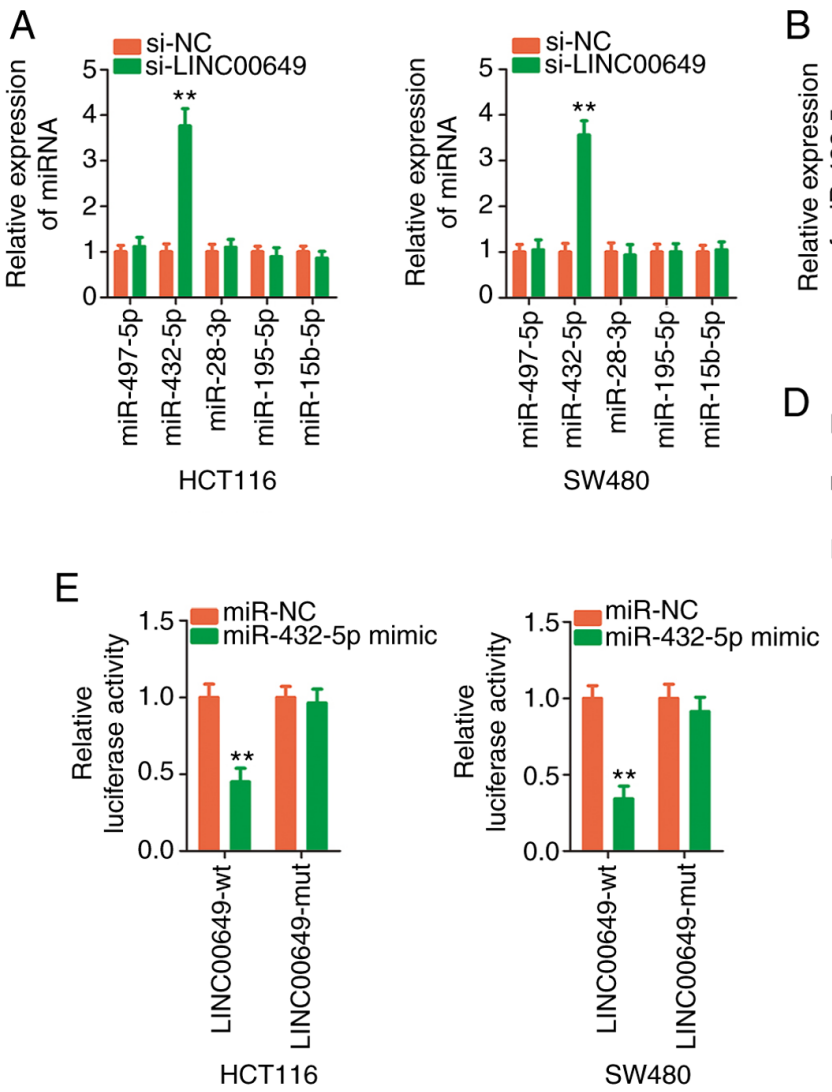

B

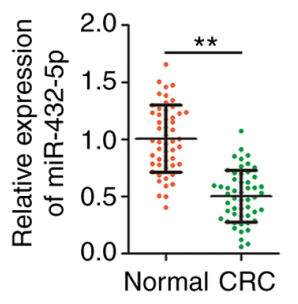

D LINC00649-wt $\quad 5^{\prime}$ miR-432-5p $\quad 3^{\prime}$

LINC00649-mut 5
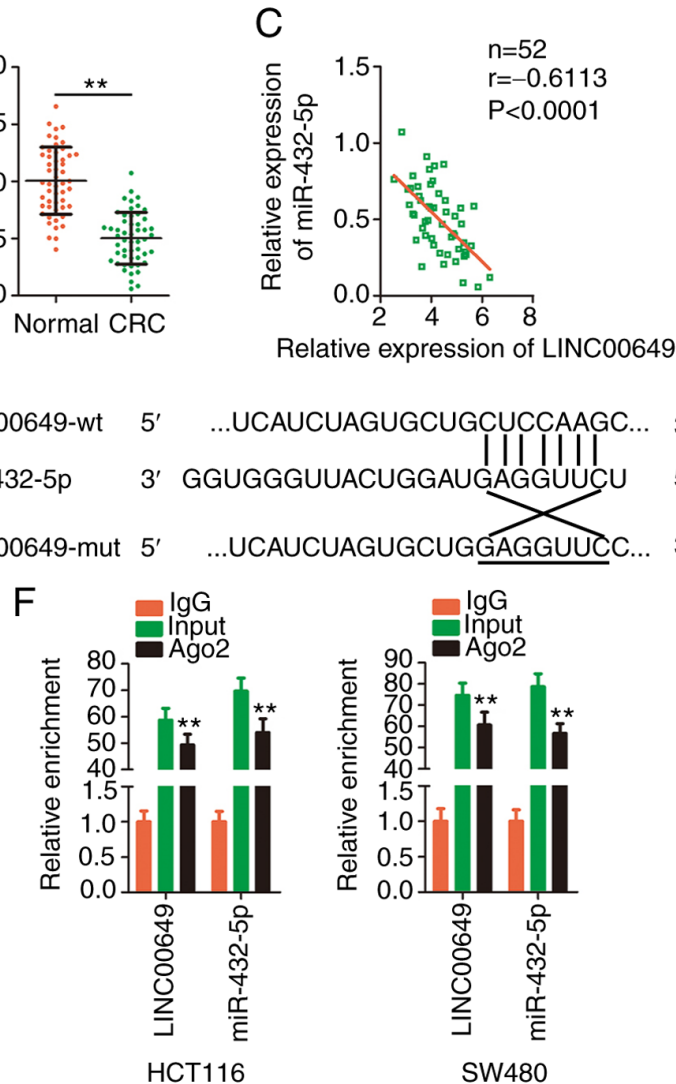

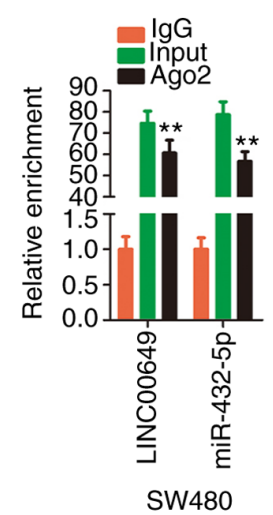

Figure 4. LINC00649 acts as a sponge of miR-432-5p. (A) The levels of five candidates were detected in CRC cells when LINC00649 was knocked down. ${ }^{* *} \mathrm{P}<0.01$ vs. si-NC. (B) miR-432-5p level in CRC tissues was explored by reverse transcription-quantitative PCR. ${ }^{* *} \mathrm{P}<0.01$. (C) Pearson's correlation analysis determined the relationship between LINC00649 and miR-432-5p levels. (D) The putative binding site between LINC00649 and miR-432-5p. (E) LINC00649-wt or LINC00649-mut alongside miR-432-5p mimic or miR-NC were co-transfected into CRC cells, followed by the quantification of luciferase activity. ${ }^{* *} \mathrm{P}<0.01$ vs. miR-NC. (F) The interaction between LINC00649 and miR-432-5p in CRC cells was further confirmed by RIP assay. ${ }^{* *} \mathrm{P}<0.01$ vs. IgG. LINC00649, long intergenic nonprotein coding RNA 649; miR, microRNA; CRC, colorectal cancer; wt, wild-type; mut, mutant; RIP, RNA immunoprecipitation; si-, small interfering; NC, negative control.

principally distributed in CRC cell cytoplasm (Fig. 3A). Thus, LINC00649 may function in a ceRNA manner. starBase 3.0 was applied to predict the target of LINC00649. In total, 12 miRNAs, including miR-28-3p, miR-16-5p, miR-15a-5p, miR-15b-5p, miR-195-5p, miR-424-5p, miR-497-5p, miR-432-5p, miR-1323, miR-4766-5p, miR-6838-5p and miR-548o-5p, contained complementary sequences to LINC00649. Utilizing TCGA database, miR-497-5p, miR-432-5p, miR-28-3p, miR-195-5p and miR-15b-5p were revealed to be downregulated in CRC (Fig. 3B-F); hence, the five miRNAs were selected for subsequent experimental confirmation.

Following LINC00649 knockdown in CRC cells, their expression was detected via RT-qPCR. The expression level of miR-432-5p was significantly increased by LINC00649 deficiency, whereas the expression of other candidates was unaltered in CRC cells upon silencing of LINC00649 (Fig. 4A). Furthermore, miR-432-5p exhibited relatively lower expression in CRC tissues (Fig. 4B) and presented a negative expression correlation with LINC00649 (Fig. 4C). The direct binding between LINC00649 and miR-432-5p (Fig. 4D) was then confirmed by employing a luciferase reporter assay. Overexpressed miR-432-5p decreased the luciferase activity of LINC00649-wt, whereas the suppression was counteracted when the binding site was mutated (Fig. 4E). As evidenced by RIP, LINC00649 and miR-432-5p were both enriched in Ago2-containing beads in comparison with IgG-containing beads (Fig. 4F). Briefly, LINC00649 sponged miR-432-5p in CRC cells.

miR-432-5p directly targets and decreases HDGF in CRC cells. Transfection with miR-432-5p mimic caused a significant upregulation of miR-432-5p (Fig. 5A). Exogenous miR-432-5p expression decreased CRC cell proliferation (Fig. 5B) and facilitated cell apoptosis (Fig. 5C). Cell migration and invasion (Fig. 5D and E) were restricted in CRC cells after miR-432-5p mimic transfection. As predicted by bioinformatics analysis, a potential binding site was revealed between miR-432-5p and the HDGF 3'UTR (Fig. 5F). Next, to confirm the prediction, a luciferase reporter assay was performed, and the results revealed that the luciferase activity of HDGF-wt, but not HDGF-mut, was attenuated by miR-432-5p (Fig. 5G). The regulatory activities of miR-432-5p overexpression on HDGF expression were then determined. HDGF expression was clearly downregulated in CRC cells when miR-432-5p was overexpressed (Fig. 5H and I). Collectively, these results confirmed HDGF as a downstream target of miR-432-5p.

si-LINC00649-induced effects on CRC cells are dependent on the miR-432-5p/HDGF axis. To assess the association 
A

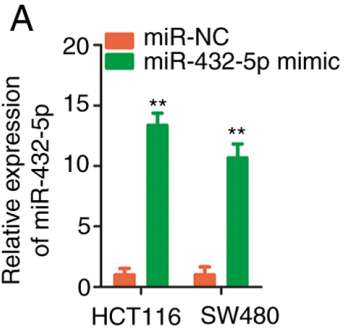

C
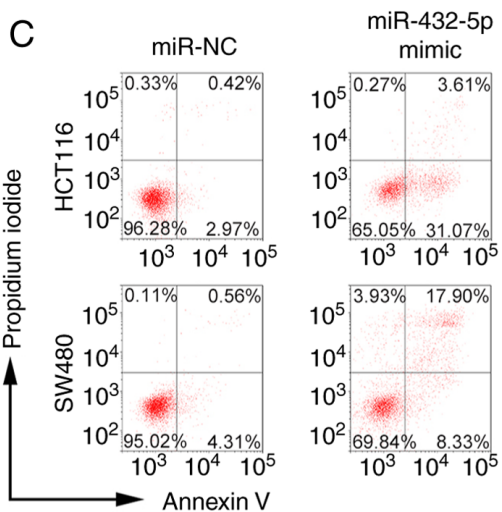

B

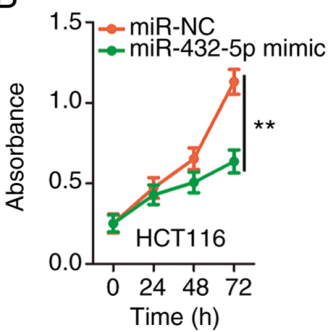

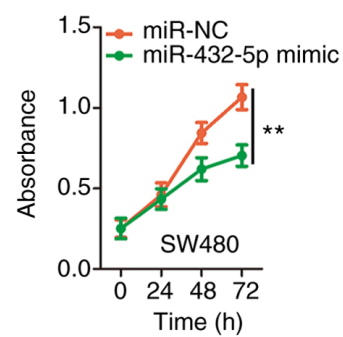

Time (h)
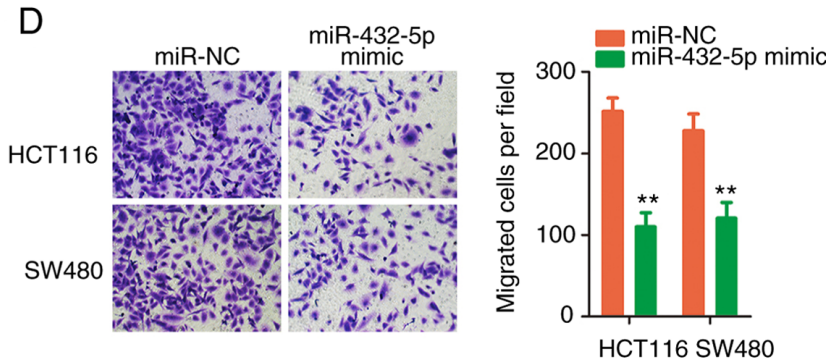

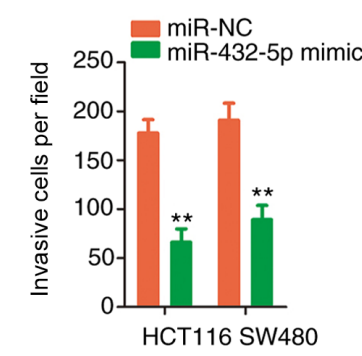

$\mathrm{F}$

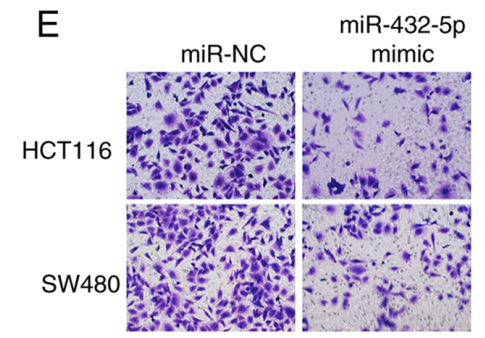

HDGF-wt $\quad 5^{\prime}$...UACUCACUUUUCCCACUCCAAGC... $3^{\prime}$

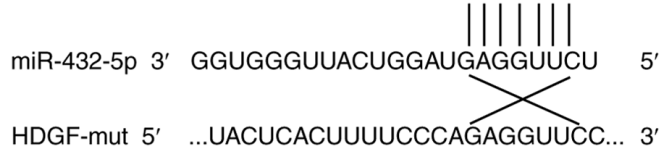

G
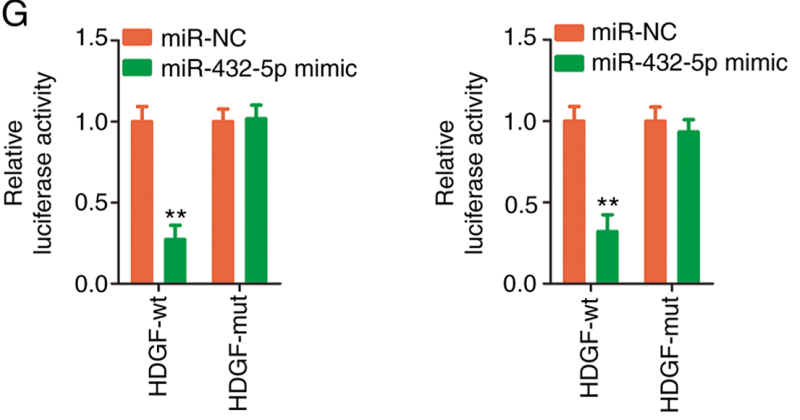

$\mathrm{H}$

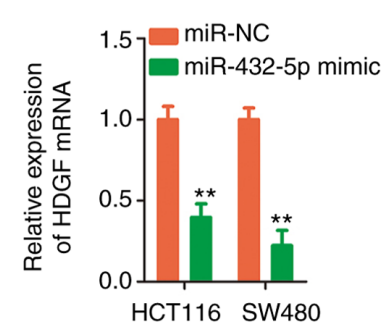

HCT116
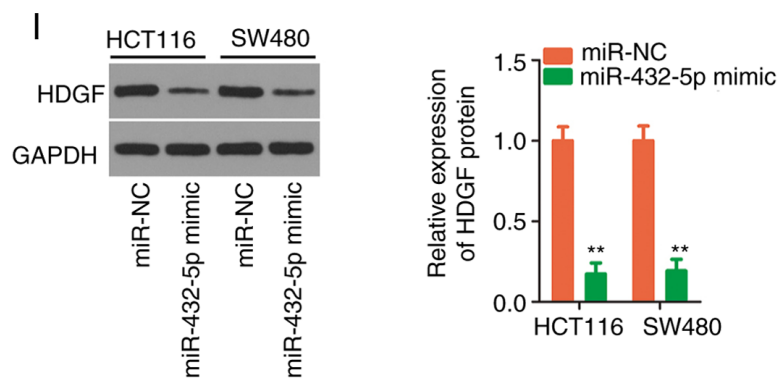

Figure 5. Confirmation of HDGF as a target of miR-432-5p. (A) RT-qPCR was employed to gauge the transfection efficiency of miR-432-5p mimic. (B and C) The proliferation and apoptosis of CRC cells after miR-432-5p overexpression were examined by Cell Counting Kit-8 assay and flow cytometric analysis, respectively. (D and E) Cell motility was analysed by Transwell cellular migration and invasion assays in CRC cells upon miR-432-5p overexpression (magnification, x200). (F) The target sequences between miR-432-5p and the HDGF 3'UTR were predicted by bioinformatics analysis. (G) Luciferase activity was quantified in CRC cells after being transfected with miR-432-5p mimic or miR-NC and HDGF-wt or HDGF-mut. (H and I) The measurement of HDGF in miR-432-5p overexpressed-CRC cells was performed by applying RT-qPCR and western blotting. ${ }^{* *} \mathrm{P}<0.01$ vs. miR-NC. HDGF, hepatoma-derived growth factor; miR, microRNA; RT-qPCR, reverse transcription-quantitative PCR; CRC, colorectal cancer; UTR, untranslated region; NC, negative control; wt, wild-type; mut, mutant. 
A

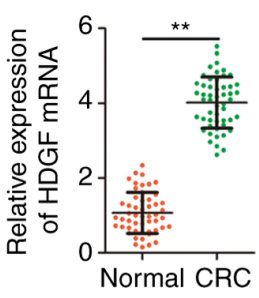

B

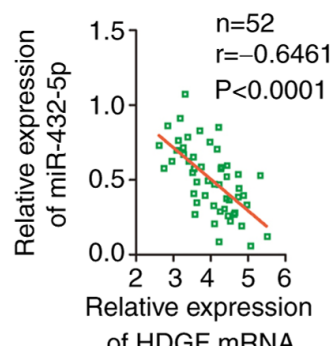

C

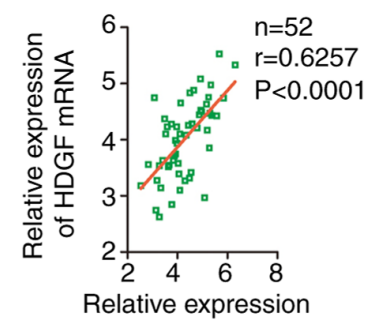

of LINC00649
D

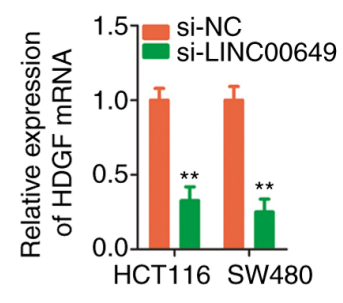

E

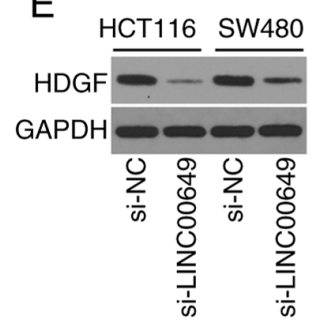

G
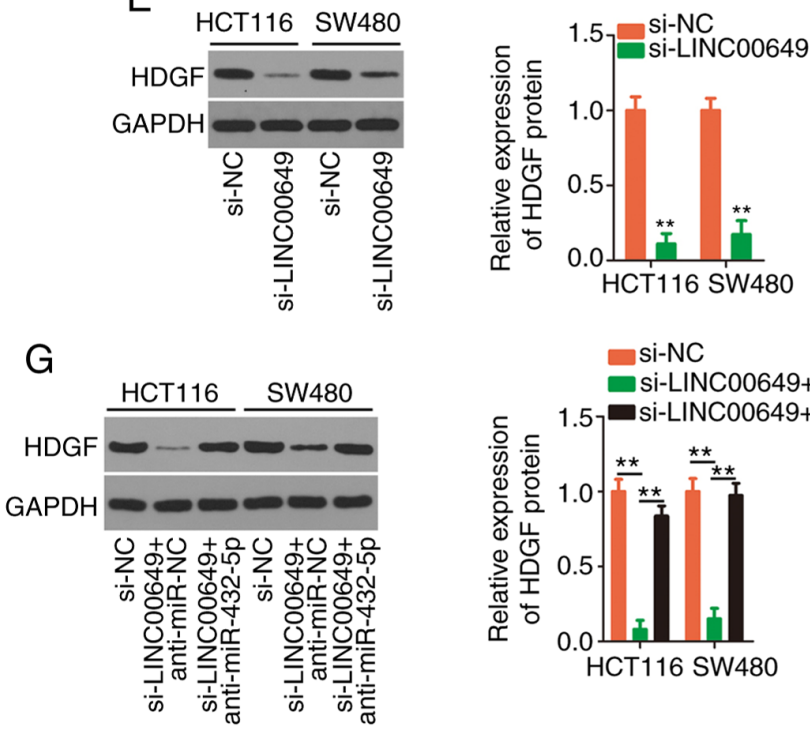

$\mathrm{F}$
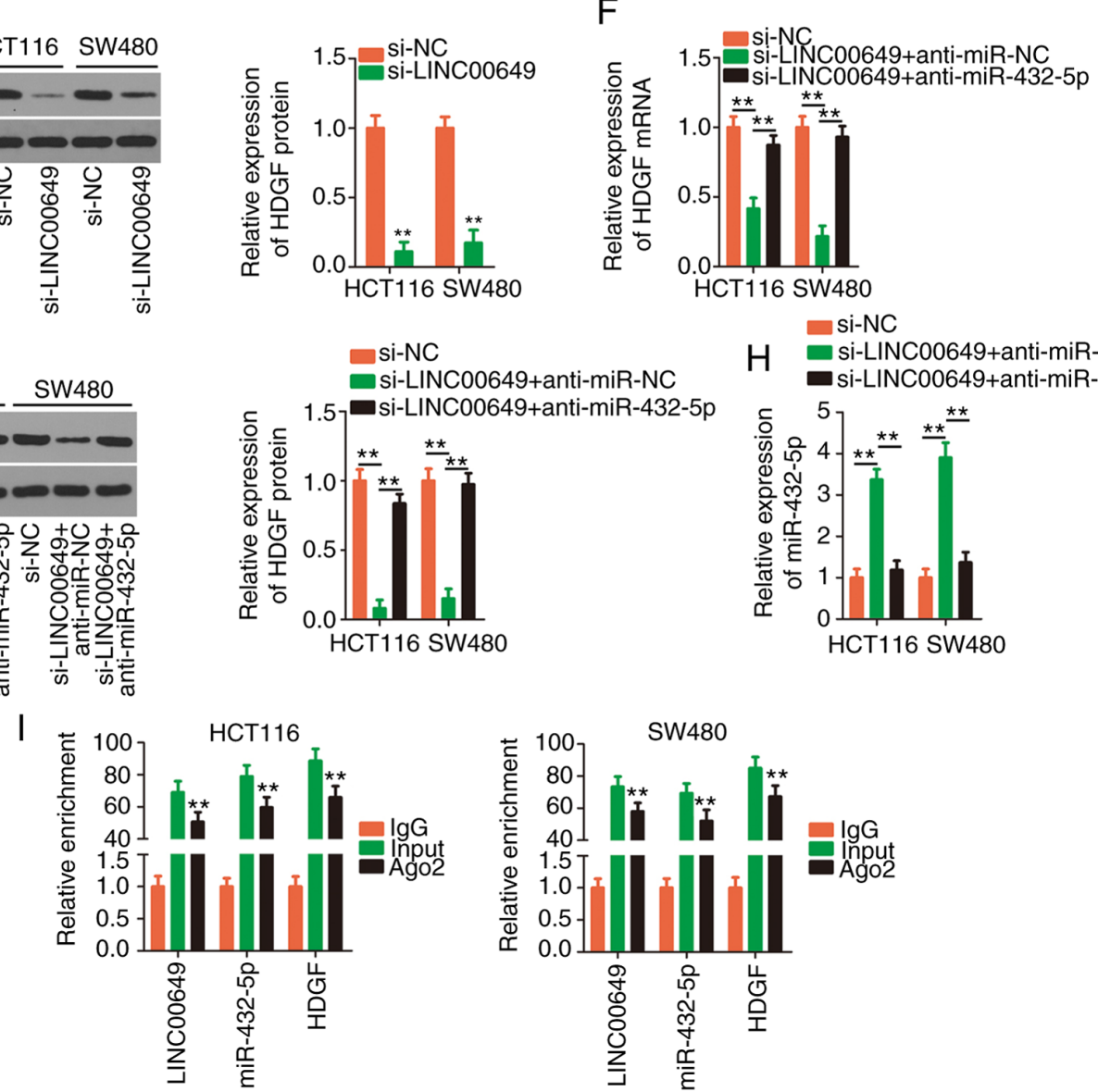

Figure 6. LINC00649 upregulates HDGF expression in CRC cells by adjusting miR-432-5p. (A) Detection of HDGF expression in CRC tissues was performed via RT-qPCR. ${ }^{* *} \mathrm{P}<0.01$. (B) Pearson's correlation analysis verified the negative expression correlation between HDGF and miR-432-5p. (C) The positive expression correlation between LINC00649 and HDGF. (D and E) RT-qPCR and western blotting detected HDGF expression in CRC cells when LINC00649 was silenced. ${ }^{* *} \mathrm{P}<0.01$ vs. si-NC. (F and G) si-LINC00649 alongside anti-miR-432-5p or anti-miR-NC was injected into CRC cells, followed by the detection of HDGF expression. (H) The change in miR-432-5p expression in the aforementioned cells was analysed by RT-qPCR. ${ }^{* *} \mathrm{P}<0.01$. (I) RIP assays confirmed the target binding among LINC00649, miR-432-5p and HDGF. ${ }^{* *} \mathrm{P}<0.01$ vs. IgG. LINC00649, long intergenic nonprotein coding RNA 649; HDGF, hepatoma-derived growth factor; CRC, colorectal cancer; miR, microRNA; RT-qPCR, reverse transcription-quantitative PCR; RIP, RNA immunoprecipitation; si-, small interfering; NC, negative control; Ago2, Argonaute-2.

between LINC00649, miR-432-5p and HDGF, Pearson's correlation analysis was applied, and the results revealed that highly expressed HDGF (Fig. 6A) in CRC tissues was inversely correlated with miR-432-5p (Fig. 6B). The positive expression correlation between LINC00649 and HDGF in CRC tissues was significant (Fig. 6C). Next, silencing of LINC00649 significantly decreased HDGF expression (Fig. 6D and E), which could be restored in CRC cells after anti-miR-432-5p co-transfection (Fig. 6F and G). Additionally, inhibiting miR-432-5p reversed the stimulatory effect of si-LINC00649 on miR-432-5p expression in CRC cells (Fig. 6H). RIP assays revealed that, compared with the $\mathrm{IgG}$ group, the three RNAs, including LINC00649, miR-432-5p and HDGF (Fig. 6I), were all enriched in Ago2-containing beads, implying that they coexisted in the RNA induction-silencing complex. The aforementioned results confirmed that LINC00649 could sponge miR-432-5p in CRC cells and subsequently increase HDGF expression.

Given primarily the obtained data, it was hypothesized that the miR-432-5p/HDGF axis is necessary for the effects of LINC00649 in CRC cells. Rescue experiments were performed to corroborate this theory. Prior to transfection, the efficiency of anti-miR-432-5p and pc-HDGF transfection was confirmed (Fig. 7A and B). The proliferative activity of CRC 
A

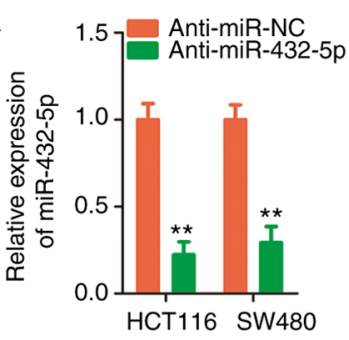

C $\rightarrow$ si-NC

- si-LINC00649+anti-miR-NC

^ si-LINC00649+anti-miR-432-5p

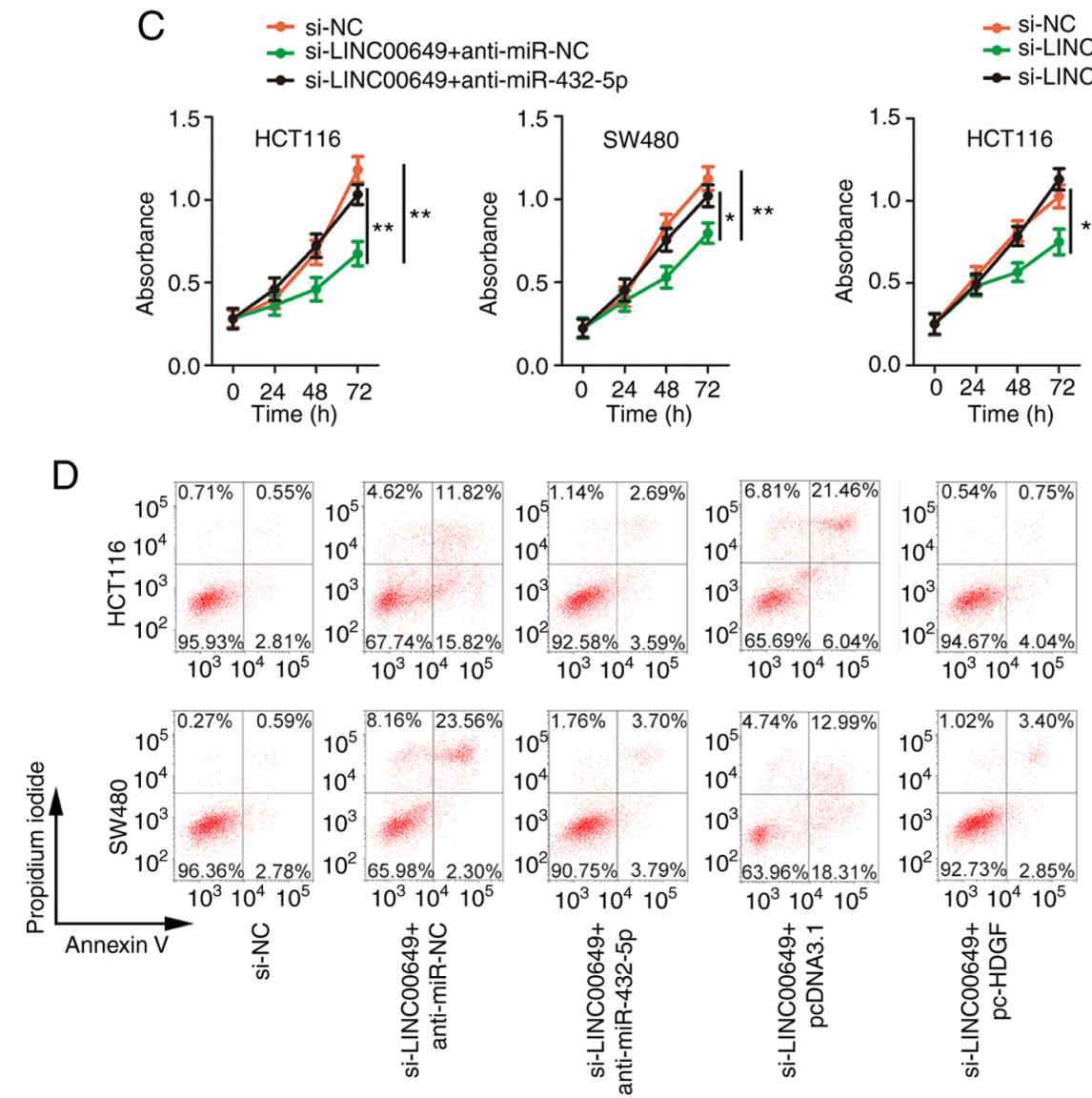

B

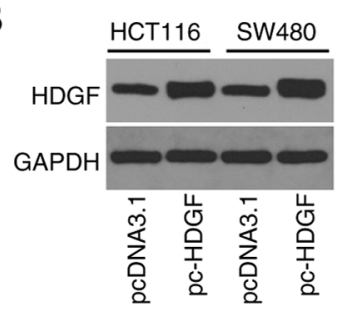

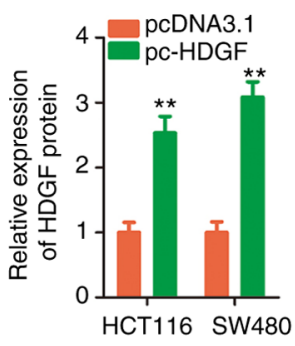

$\approx$ si-NC

- si-LINC00649+pcDNA3.1

$\rightarrow$ si-LINC00649+pc-HDGF
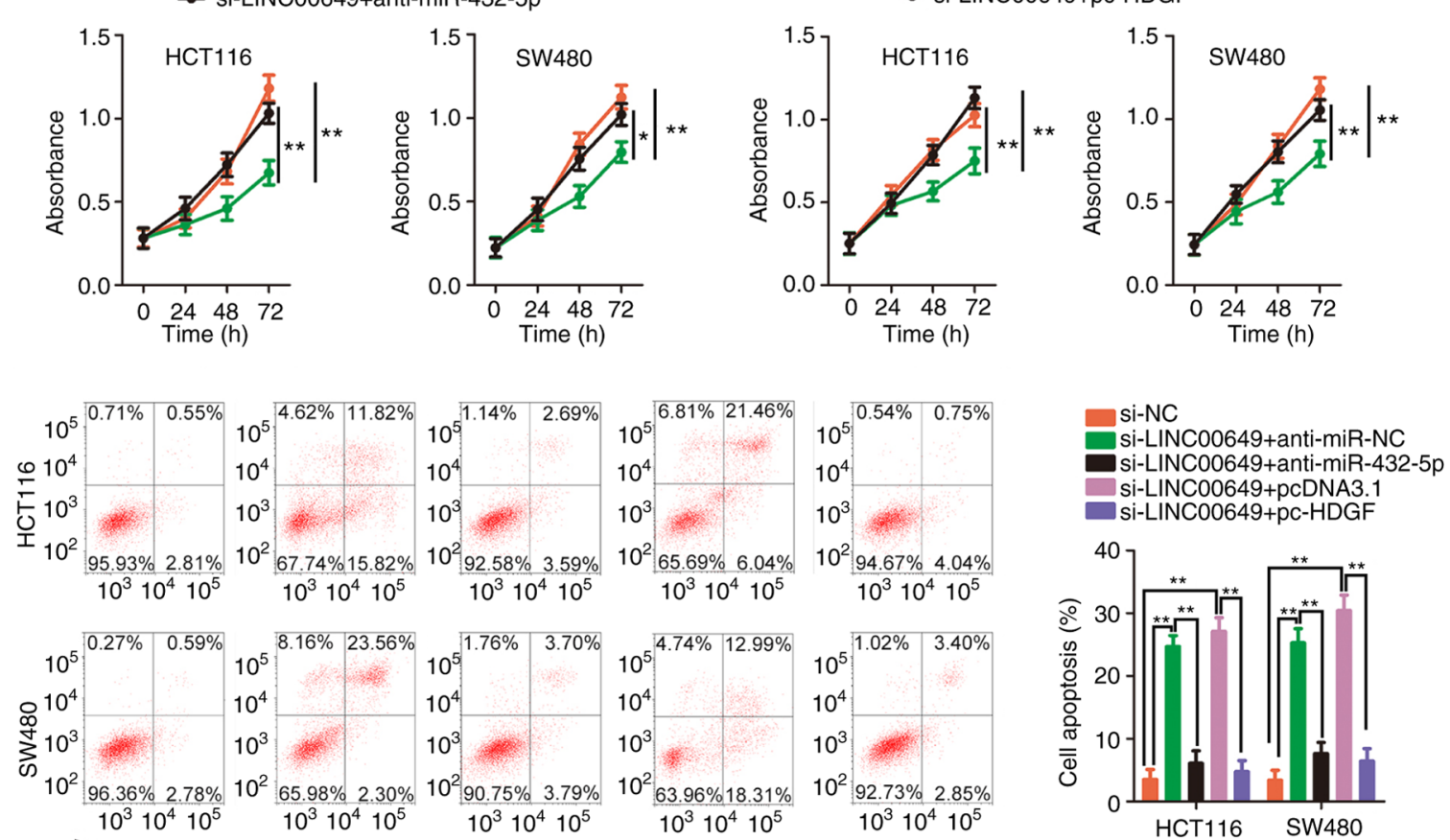

Figure 7. Downregulation of miR-432-5p or introduction of HDGF relieves the si-LINC00649-induced influences on cell proliferation and apoptosis in CRC. (A) The silencing efficiency of anti-miR-432-5p was examined by reverse transcription-quantitative PCR. ${ }^{* *} \mathrm{P}<0.01$ vs. Anti-miR-NC. (B) The overexpression efficiency of the pc-HDGF plasmid was verified by western blotting. ${ }^{* *} \mathrm{P}<0.01$ vs. pcDNA3.1. (C and D) LINC00649-silenced CRC cells were co-transfected with anti-miR-432-5p or pc-HDGF, and cell proliferation and apoptosis determination was performed. ${ }^{*} \mathrm{P}<0.05$ and ${ }^{* *} \mathrm{P}<0.01$. miR, microRNA; HDGF, hepatoma-derived growth factor; si-, small interfering; LINC00649, long intergenic nonprotein coding RNA 649; CRC, colorectal cancer; NC, negative control; pc-HDGF, pcDNA3.1-HDGF.

cells was inhibited by LINC00649 downregulation, which was mitigated by miR-432-5p inhibition or HDGF upregulation (Fig. 7C). Additionally, the si-LINC00649-induced effect on cell apoptosis was counteracted by co-transfection of anti-miR-432-5p or pc-HDGF (Fig. 7D). Furthermore, interference with LINC00649 hindered cell migration (Fig. 8A) and invasion (Fig. 8B) properties, whereas these effects were abated by the addition of anti-miR-432-5p or pc-HDGF. In summary, LINC00649 played a carcinogenic role in CRC cells via the miR-432-5p/HDGF axis.

Depletion of LINC00649 attenuates tumour growth in vivo. To demonstrate the effect of LINC00649 on in vivo tumour growth, sh-LINC00649 was designed, and HCT 116 cells stably expressing sh-LINC00649 were established (Fig. 9A).
The tumour diameter (Fig. 9B) and volume (Fig. 9C) in the sh-LINC00649 group were evidently lower than sh-NC group. Additionally, the weight of tumours in sh-LINC00649 group was decreased (Fig. 9D). Moreover, sh-LINC00649-injected tumours presented decreased LINC00649 (Fig. 9E) and increased miR-432-5p levels (Fig. 9F). Furthermore, HDGF protein was reduced in tumours obtained from the sh-LINC00649 group (Fig. 9G). Collectively, downregulation of LINC00649 inhibited CRC growth in vivo.

\section{Discussion}

As tumour molecular biology progresses, extensive studies have confirmed that IncRNAs have a significant effect on the oncogenicity of CRC (21-23). With the universal application 

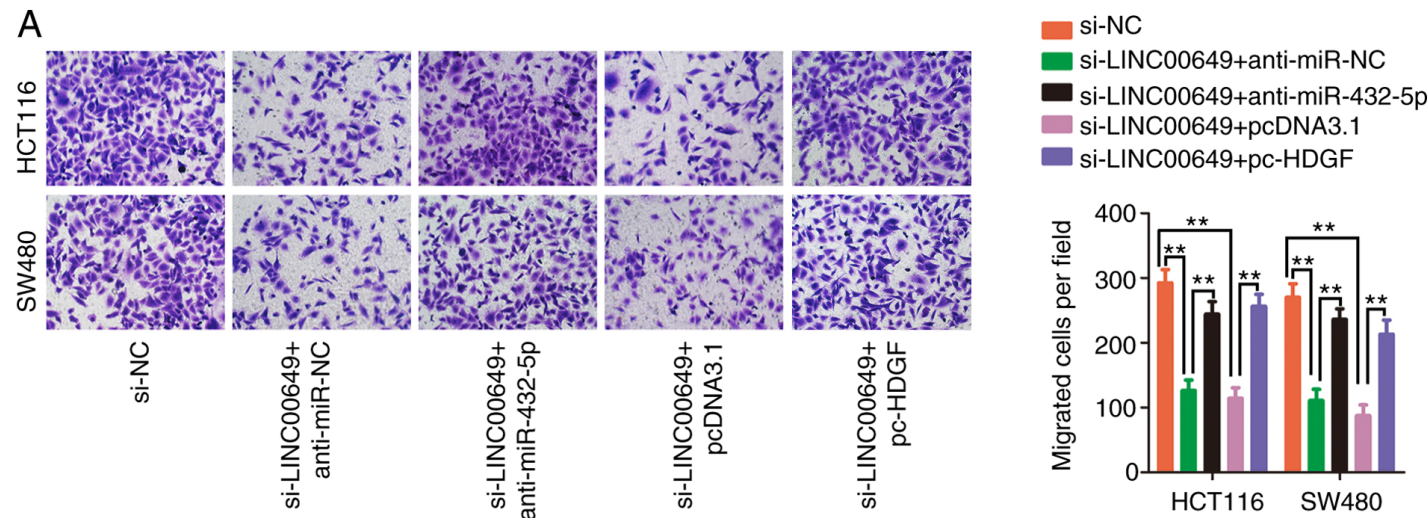

B
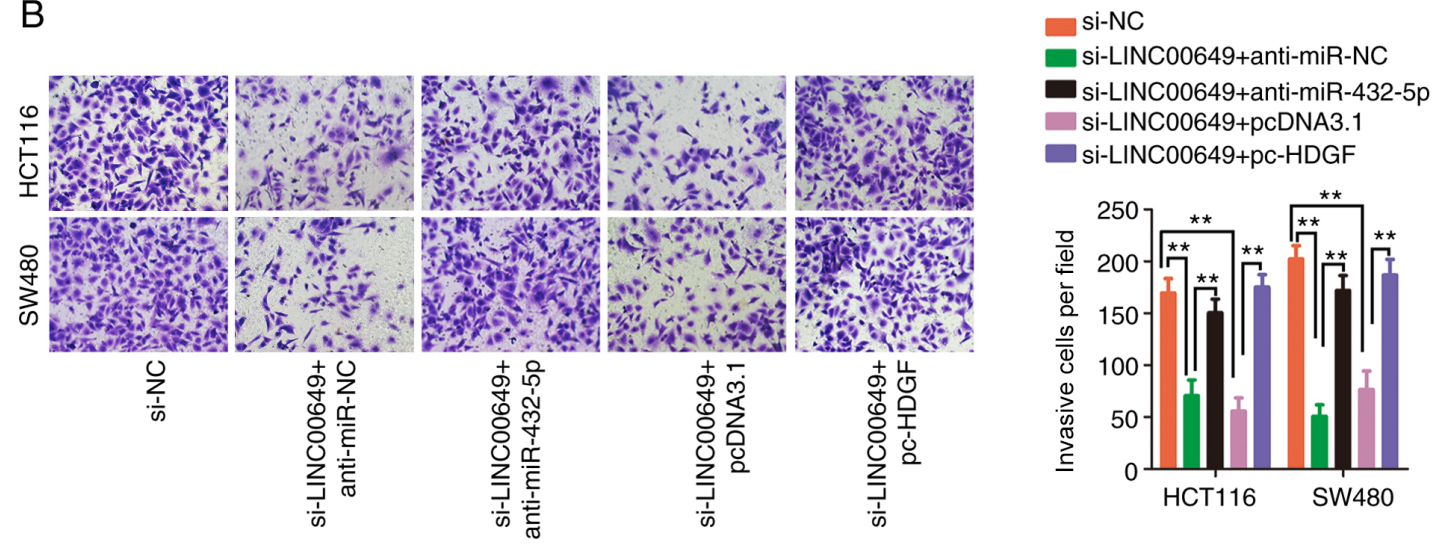

Figure 8. Effects of LINC00649 silencing on CRC cell motility are counteracted by miR-432-5p downregulation or HDGF reintroduction. (A and B) The LINC00649-silenced CRC cells were co-transfected with anti-miR-432-5p or pc-HDGF, and subjected to Transwell cellular migration and invasion assays for motility assessment (magnification, $\mathrm{x} 200$ ). ${ }^{* *} \mathrm{P}<0.01$. LINC00649, long intergenic nonprotein coding RNA 649; CRC, colorectal cancer; miR, microRNA; HDGF, hepatoma-derived growth factor; si-, small interfering; NC, negative control; pc-HDGF, pcDNA3.1-HDGF.

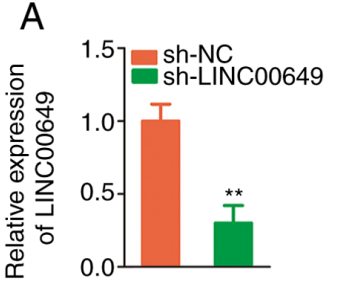

$E$

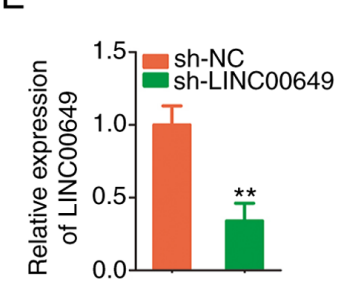

B

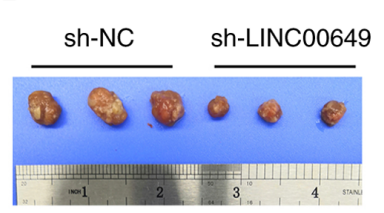

$\mathrm{F}$

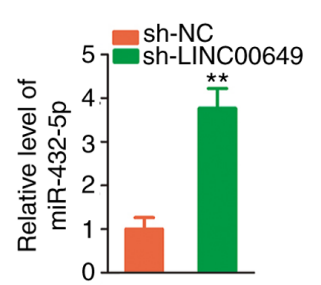

C

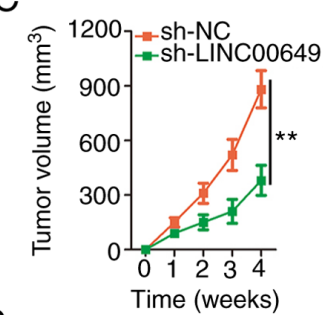

G

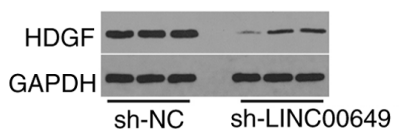

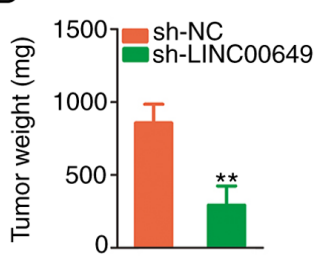

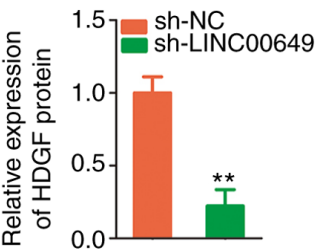

Figure 9. Effect of sh-LINC00649 on the tumour growth in vivo. (A) The efficiency of sh-LINC00649 in HCT 116 cells was confirmed via reverse transcription-quantitative PCR. (B) Representative images of tumour xenografts. (C) The tumour volume was monitored at 7, 14, 21 and 28 days after tumour cell injection. Tumour growth curves were constructed according to the recorded data. (D) The tumour weight was measured in tumour xenografts. (E-G) Assessment of LINC00649, HDGF and miR-432-5p levels in tumour xenografts. ${ }^{* *} \mathrm{P}<0.01$ vs. sh-NC. sh-, short hairpin; LINC00649, long intergenic nonprotein coding RNA 649; NC, negative control; HDGF, hepatoma-derived growth factor; miR, microRNA.

of high-throughput sequencing, various lncRNAs have been identified to be differentially expressed in CRC (10). Nevertheless, numerous IncRNAs have yet to be investigated, and the mechanisms involving lncRNAs in the pathogenesis of CRC are not completely understood. Thus, the present study was initiated to offer conclusive evidence regarding the involvement of LINC00649 in CRC and the crosstalk between LINC00649 and miR-432-5p.

LINC00649 is expressed at a low level in acute myeloid leukaemia (17). Patients with acute myeloid leukaemia 
manifesting a low LINC00649 level have shorter progression-free survival and overall survival than patients with a high LINC00649 level (17). However, the expression and contribution of LINC00649 in CRC have yet to be described. According to TCGA database data, it was confirmed that LINC00649 was overexpressed in both COAD and READ relative to normal tissues. In line with this tendency, the expression level of LINC00649 was revealed to be increased in CRC tissues of our own cohort. More importantly, the aberrant expression of LINC00649 exhibited an inverse association with the prognosis of patients with CRC. Functionally, loss of LINC00649 hindered the aggressive properties of CRC cells. Therefore, the expression status and functions of LINC00649 in human cancers exhibited tissue specificity.

Mechanistically, lncRNAs exercise their biological tasks by working with different molecules (24). At the transcriptional level, lncRNAs can epigenetically silence mRNAs or degrade proteins (25). At the post-transcriptional level, ceRNA crosstalk among lncRNAs, miRNAs and mRNAs has been recognized and has attracted increasing interest (26). The cytoplasmic localization of lncRNAs can operate as 'miRNA sponges' and competitively bind to certain miRNAs, thereby decreasing the targets of miRNAs from silencing or translational inhibition (27). Clarification of the subcellular localization of LINC00649 revealed that it was located in the cytosol of CRC cells. Accordingly, LINC00649 may function as a ceRNA in CRC.

Predictably, it was revealed that miR-432-5p may play a role as a miRNA sponge of LINC00649. Additionally, the present study revealed that the silencing of LINC00649 distinctly improved miR-432-5p expression in CRC cells. Next, luciferase reporter assays and Ago2 RIP assays confirmed that LINC00649 physically interacted and directly bound with miR-432-5p in CRC, thus sequestering miR-432-5p at the molecular level. Subsequent experiments were performed to determine the target mRNA of miR-432-5p to construct a complete lncRNA/miRNA/mRNA pathway. The mechanistic investigation identified HDGF as a direct downstream functional target of miR-432-5p. In CRC, a negative expression correlation was confirmed between LINC00649 and miR-432-5p, while LINC00649 and HDGF levels presented a positive correlation. Furthermore, HDGF was under the positive control of LINC00649 in CRC cells, which occurred through sponging miR-432-5p. Collectively, a novel ceRNA theory consisting of LINC00649, miR-432-5p and HDGF was identified in CRC.

To date, multiple studies have highlighted the involvement of miR-432-5p in several human cancers (28-30). Our present study also attempted to reveal the importance of miR-432-5p in CRC. A notable downregulation of miR-432-5p in CRC was revealed in both the TCGA database and our own cohort, which was in line with a recent study (31). miR-432-5p has anti-oncogenic activities in CRC, and HDGF was confirmed as a downstream target of miR-432-5p. HDGF was validated as an essential contributor to the malignancy of CRC (32-35). Numerous miRNAs, including miR-93-5p (36), miR-511 (37), and miR-610 (38), have been revealed to participate in the regulation of HDGF in CRC, but it remains uncertain whether HDGF can be regulated by certain lncRNAs in CRC. In particular, compelling evidence has been presented to support that LINC00649 positively affects the expression pattern of
HDGF. Furthermore, a functional rescue assay was employed and revealed that the exogenous introduction of miR-432-5p inhibitor or HDGF overexpression plasmid partially abated the anticarcinogenic actions of LINC00649 knockdown in CRC cells. All of the aforementioned observations indicated that LINC00649 modulated the miR-432-5p/HDGF axis and thereby aggravated the oncogenicity of CRC cells.

The present study had several limitations. First, only 52 pairs of CRC tissues and adjacent normal tissues were collected, thus the sample size was too small. Furthermore, the regulatory actions of LINC00649 silencing on CRC cell proliferation, apoptosis, migration and invasion were explored in vitro; however, the effects of LINC00649 overexpression on these aggressive phenotypes were not examined. Finally, this study did not investigate the influence of LINC00649 on metastasis in vivo.

In summary, the present research demonstrated that LINC00649 played a carcinogenic role in CRC by competitively binding miR-432-5p, thus inducing increased HDGF expression. Jointly, the LINC00649/miR-432-5p/HDGF pathway may serve as an attractive target for CRC therapy.

\section{Acknowledgements}

Not applicable.

\section{Funding}

The present study was supported by The First People's Hospital of Chongqing Liangjiang New District (Chongqing, China).

\section{Availability of data and materials}

All datasets used and/or analysed during the current study are available from the corresponding author on reasonable request.

\section{Authors' contributions}

JW and XL designed the research. JB, XB, JW and XL conducted the experiments. XL analysed the obtained data. JW and XL wrote the manuscript. XL supervised the junior staff. All authors read and approved the final manuscript. JW and XL confirm the authenticity of all raw data.

\section{Ethics approval and consent to participate}

The present study was authorized (approval no. EC-FPHCQL ND-20150815) by the Ethics Committee of The First People's Hospital of Chongqing Liangjiang (Chongqing, China). Written informed consent was provided by all participants. The animal study was authorized (approval no. IACUC-FPHCQLND-2019 0601) by the Institutional Animal Care and Use Committee of The First People's Hospital of Chongqing Liangjiang.

\section{Patient consent for publication}

Not applicable.

\section{Competing interests}

The authors declare that they have no competing interests. 


\section{References}

1. Bray F, Ferlay J, Soerjomataram I, Siegel RL, Torre LA and Jemal A: Global cancer statistics 2018: GLOBOCAN estimates of incidence and mortality worldwide for 36 cancers in 185 countries. CA Cancer J Clin 68: 394-424, 2018.

2. Siegel RL, Miller KD, Goding Sauer A, Fedewa SA, Butterly LF, Anderson JC, Cercek A, Smith RA and Jemal A: Colorectal cancer statistics, 2020. CA Cancer J Clin 70: 145-164, 2020.

3. Mitry E, Guiu B, Cosconea S, Jooste V, Faivre J and Bouvier AM: Epidemiology, management and prognosis of colorectal cancer with lung metastases: A 30-year population-based study. Gut 59: $1383-1388,2010$.

4. Dekker E and Rex DK: Advances in CRC prevention: Screening and surveillance. Gastroenterology 154: 1970-1984, 2018.

5. Tol J, Koopman M, Cats A, Rodenburg CJ, Creemers GJ, Schrama JG, Erdkamp FL, Vos AH, van Groeningen CJ, Sinnige HA, et al: Chemotherapy, bevacizumab, and cetuximab in metastatic colorectal cancer. N Engl J Med 360: 563-572, 2009.

6. Ponting CP, Oliver PL and Reik W: Evolution and functions of long noncoding RNAs. Cell 136: 629-641, 2009.

7. Kung JT, Colognori D and Lee JT: Long noncoding RNAs: Past, present, and future. Genetics 193: 651-669, 2013.

8. Quinn JJ and Chang HY: Unique features of long non-coding RNA biogenesis and function. Nat Rev Genet 17: 47-62, 2016.

9. Schwarzmueller L, Bril O, Vermeulen L and Léveillé N: Emerging role and therapeutic potential of lncRNAs in colorectal cancer. Cancers (Basel) 12: 3843, 2020.

10. Talebi A, Azizpour M, Agah S, Masoodi M, Mobini GR and Akbari A: The relevance of long noncoding RNAs in colorectal cancer biology and clinical settings. J Cancer Res Ther 16 (Suppl): S22-S33, 2020.

11. Srinivasan S, Selvan ST, Archunan G, Gulyas B and Padmanabhan P: MicroRNAs -the next generation therapeutic targets in human diseases. Theranostics 3: 930-942, 2013.

12. Shukla GC, Singh J and Barik S: MicroRNAs: Processing, maturation, target recognition and regulatory functions. Mol Cell Pharmacol 3: 83-92, 2011.

13. Wen XQ, Qian XL, Sun HK, Zheng LL, Zhu WQ, Li TY and Hu JP: MicroRNAs: multifaceted regulators of colorectal cancer metastasis and clinical applications. Onco Targets Ther 13 10851-10866, 2020.

14. Ahadi A: The significance of microRNA deregulation in colorectal cancer development and the clinical uses as a diagnostic and prognostic biomarker and therapeutic agent. Noncoding RNA Res 5: 125-134, 2020

15. Weidle UH, Brinkmann U and Auslaender S: microRNAs and corresponding targets involved in metastasis of colorectal cancer in preclinical in vivo models. Cancer Genomics Proteomics 17: 453-468, 2020

16. Wang L,Cho KB, Li Y, Tao G, Xie Z and Guo B: Long noncoding RNA (lncRNA)-mediated competing endogenous RNA networks provide novel potential biomarkers and therapeutic targets for colorectal cancer. Int J Mol Sci 20: 5758, 2019.

17. Guo C, Gao YY, Ju QQ, Zhang CX, Gong M and Li ZL: LINC00649 underexpression is an adverse prognostic marker in acute myeloid leukemia. BMC Cancer 20: 841, 2020.

18. He M, Lin Y and Xu Y: Identification of prognostic biomarkers in colorectal cancer using a long non-coding RNA-mediated competitive endogenous RNA network. Oncol Lett 17: 2687-2694, 2019

19. Livak KJ and Schmittgen TD: Analysis of relative gene expression data using real-time quantitative PCR and the 2(-Delta Delta C(T)) method. Methods 25: 402-408, 2001.

20. Deng M, Bragelmann J, Schultze JL and Perner S: Web-TCGA: An online platform for integrated analysis of molecular cancer data sets. BMC Bioinformatics 17: 72, 2016.

21. Hon KW, Abu N, Ab Mutalib NS and Jamal R: miRNAs and $\operatorname{lncRNAs}$ as predictive biomarkers of response to FOLFOX therapy in colorectal cancer. Front Pharmacol 9: 846, 2018 .
22. Rivandi M, Pasdar A, Hamzezadeh L, Tajbakhsh A, Seifi S, Moetamani-Ahmadi M, Ferns GA and Avan A: The prognostic and therapeutic values of long noncoding RNA PANDAR in colorectal cancer. J Cell Physiol 234: 1230-1236, 2019.

23. Wei L, Wang X, Lv L, Zheng Y, Zhang N and Yang M: The emerging role of noncoding RNAs in colorectal cancer chemoresistance. Cell Oncol (Dordr) 42: 757-768, 2019.

24. Gao N, Li Y, Li J, Gao Z, Yang Z, Li Y, Liu H and Fan T: Long non-coding RNAs: The regulatory mechanisms, research strategies, and future directions in cancers. Front Oncol 10: 598817, 2020.

25. Samimi H, Sajjadi-Jazi SM, Seifirad S, Atlasi R, Mahmoodzadeh H, Faghihi MA and Haghpanah V: Molecular mechanisms of long non-coding RNAs in anaplastic thyroid cancer: A systematic review. Cancer Cell International 20: 352 , 2020.

26. Qi X, Zhang DH, Wu N, Xiao JH, Wang X and Ma W: ceRNA in cancer: Possible functions and clinical implications. J Med Genet 52: 710-718, 2015.

27. Ergun S and Oztuzcu S: Oncocers: ceRNA-mediated cross-talk by sponging miRNAs in oncogenic pathways. Tumour Biol 36: 3129-3136, 2015

28. Das S and Bhattacharyya NP: Heat shock factor 1 regulates hsa-miR-432 expression in human cervical cancer cell line. Biochem Biophys Res Commun 453: 461-466, 2014.

29. Jiang N, Chen WJ, Zhang JW, Xu C, Zeng XC, Zhang T, Li Y and Wang GY: Downregulation of miR-432 activates Wnt/ $\beta$-catenin signaling and promotes human hepatocellular carcinoma proliferation. Oncotarget 6: 7866-7879, 2015.

30. Chen L, Kong G, Zhang C, Dong H, Yang C, Song G, Guo C, Wang L and Yu H: MicroRNA-432 functions as a tumor suppressor gene through targeting E2F3 and AXL in lung adenocarcinoma. Oncotarget 7: 20041-20053, 2016.

31. Luo M, Hu Z, Kong Y and Li L: MicroRNA-432-5p inhibits cell migration and invasion by targeting CXCL5 in colorectal cancer. Exp Ther Med 21: 301, 2021.

32. Lian J, Tang J, Shi H, Li H, Zhen T, Xie W, Zhang F, Yang Y and Han A: Positive feedback loop of hepatoma-derived growth factor and beta-catenin promotes carcinogenesis of colorectal cancer. Oncotarget 6: 29357-29374, 2015.

33. Liao F, Liu M,Lv L and Dong W: Hepatoma-derived growth factor promotes the resistance to anti-tumor effects of nordihydroguaiaretic acid in colorectal cancer cells. Eur J Pharmacol 645: 55-62, 2010.

34. Hu TH, Lin JW, Chen HH, Liu LF, Chuah SK and Tai MH: The expression and prognostic role of hepatoma-derived growth factor in colorectal stromal tumors. Dis Colon Rectum 52: 319-326, 2009.

35. Liao F, Dong W and Fan L: Apoptosis of human colorectal carcinoma cells is induced by blocking hepatoma-derived growth factor. Med Oncol 27: 1219-1226, 2010.

36. Hong YG, Huang ZP, Liu QZ, E JF, Gao XH, Xin C, Zhang W, Li P and Hao LQ: MicroRNA-95-3p inhibits cell proliferation and metastasis in colorectal carcinoma by HDGF. Biomed J 43: 163-173, 2020.

37. He S, Wang G, Ni J, Zhuang J, Zhuang S, Wang G, Ye Y and Xia W: MicroRNA-511 inhibits cellular proliferation and invasion in colorectal cancer by directly targeting hepatoma-derived growth factor. Oncol Res 26: 1355-1363, 2018.

38. Sun B, Gu X, Chen Z and Xiang J: MiR-610 inhibits cell proliferation and invasion in colorectal cancer by repressing hepatoma-derived growth factor. Am J Cancer Res 5: 3635-3644, 2015.

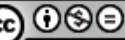

This work is licensed under a Creative Commons Attribution-NonCommercial-NoDerivatives 4.0 International (CC BY-NC-ND 4.0) License. 